\title{
O Número de Milnor
}

\author{
João Nivaldo Tomazella
}

Orientação.

Profa. Dra. Maria Aparecida Soares Ruas

Disstrtaçào apresentada ao Instituto de Ciéncias .11atemáticas de Sâo Carlos - lSP. como parte dos requisitos para obtençâo do título de "Mestre em Ciéncias - Área: Matemática". 
A minha famitia 


\section{Agradecimentos}

À Cidinha, pela orientaçào, dedicaçào e pelo incentivo à nossa iniciaçào a pesquisa.

Ao professor Janey pelas várias discussòes esclarecedoras e pela fundamental ajuda na demonstraçào do Teorema 1.i.

Aos professores Biasi e Ires pelos comentários e discussôes esclarecedoras.

A Beth e Laura. pela dedicaçào e amizade.

Aos amigos de pós-graduaçào e de república.

Ao CNPq. pelo apoio financeiro. 


\begin{abstract}
J. Milnor [1] studied the topology of complex hypersurfaces by introducing a local fibration which is associated with each isolated singular point. He showed that the fiber of the fibration has the homotopy type of a wedge of spheres. The number of spheres of this wedge is the Milnor number, which is an invariant of the topological type of the singularity.

In this dissertation we study the geometric and the algebraic characterization of the Milnor number and we obtain the equivalence between them.
\end{abstract}




\section{Resumo}

J. Milnor [1] estudou a topologia de hipersuperficies complexas introduzindo uma fibraçào local a qual está associada a singularidade isolada. Ele mostrou que a fibra da fibraçâo tem o mesmo tipo de homotopia de um bouquet de esferas. $\mathrm{O}$ número de esferas deste bouquet é o número de Milnor. o qual é um invariante do tipo topológico da singularidade.

Nesta dissertaçâo nós estudamos a caracterizaçào geométrica e algébrica do número de Milnor e obtivemos a equivalencia entre elas. 


\section{Conteúdo}

1 O NÚMERO DE MILNOR 3

1.1 O Teorema da Fibração e a Topologia da Fibra . . . . . . . . . 3

1.2 Definiçào do Número de Milnor .............

1.3 O Número de Milnor como Invariante Topológico . . . . . . . . . 10

2 Caracterização Geométrica do Número de Milnor 16

2.1 O Grau de uma Aplicaçào . . . . . . . . . . . . . 16

2.2 A Multiplicidade Gradiente . . . . . . . . . . . . . 18

3 Caracterização Algébrica do Número de Milnor 23

4 Equivalência das Definições $\quad 30$

4.1 Equivalência entre a Multiplicidade Geométrica e o Número de Milnor . . . . . . . . . . . . . . . . . . . . 30

4.2 Equivalência entre a Multiplicidade Algébrica e a Multiplicidade Gradiente . . . . . . . . . . . . . . 36

5 Cálculo do Número de Milnor 42

$\begin{array}{lr}\text { Bibliografia } & 45\end{array}$ 


\section{Introdução}

Os estudos deste trabalho se basejam nos textos: Singular Points of Complex Hypersurfaces [1] e Introdução à Topologia de Singularidades Complexas [5]. que descrevem os resultados de Milnor sobre a topologia de uma hipersuperfície complexa na vizinhança de um ponto singular isolado.

Uma hipersuperfície complexa $V$ em $\mathrm{C}^{n}$ é o conjunto $V=\left\{z \in \mathbf{C}^{n}: f(z)=0\right\}=f^{-1}(0)$ onde $f$ é uma função holomorfa.

Indiquemos por $K^{\prime}$ a intersecsào de $I^{\prime}$ com uma esfera $S_{\varepsilon}$, de rajo $\varepsilon$, centrada na origem. Os resultados básicos da teoria de Milnor são o Teorema da Fibração que estabelece que $S_{\varepsilon}-h$ é um fibrado localmente trivial sobre $S^{1}$, com fibra típica $F_{\theta}$ e os Teoremas que descrevem a topologia da fibra $F_{\theta}$.

Quando $f$ tem singularidade isolada. Milnor mostrou que cada fibra $F_{6}$ tem o mesmo tipo de homotopia de um bouquet de esferas. Este resultado permite definir um invariante topológico local de $V$. Esse invariante é o número de Milnor, $\mu$, que é o número de esferas do bouquet.

Os seguintes resultados de Lê Dũng Tráng mostram que $\mu$ é um invariante completo do tipo topológico da singularidade. Se dois germes de hipersuperfícies complexas $V=f^{-1}(0)$ e $H^{*}=g^{-1}(0)$ com singularidades isoladas sào homeomorfos. então possuem o mesmo número de Milnor. Além disso. se uma deformação $f_{t}$ de um germe $f$ tem número de Milnor $\mu\left(f_{t}\right)$ constante, entâo as varjedades $V_{t}=f_{t}^{-1}(0)$ sâo topologicamente equivalentes.

O número de Milnor admite caracterizaçôes geométricas e algébricas surpreendentes. Geometricamente. $\mu(f)$ é caracterizado como grau da aplicaçào $\frac{\partial f}{|\partial f|}: S_{\varepsilon} \rightarrow S_{1}$. Algebricamente. o número de Milnor é dado pela fórmula: $\mu(f)=\operatorname{dim} \mathcal{O}_{n} / J f$, onde $\mathcal{O}_{n}$ é o conjunto dos germes em 0 de funçôes holomorfas e $J f$, o ideal de $\mathcal{O}_{n}$ gerado pelos germes em 0 das derivadas parciais $\frac{\partial f}{\partial z_{1}}$ para $i=1, \ldots, n$.

Esta dissertação tem como objetivo o estudo das diversas caracterizaçôes do número de Milnor e a obtenção da equivalência entre elas.

No capitulo 1 enunciamos os resultados básicos do trabalho de Milnor, que sâo o Teorema da Fibração e os teoremas sobre a topologia da fibra. Além disso, demonstramos o teorema de Lê Dúng Tráng que estabelece que o número de 
Milnor é um invariante da singularidade.

No capitulo 2 introduzimos a multiplicidade gradiente. $\mu_{g}(f)$, de um germe $f:\left(\mathbf{C}^{n}, 0\right) \rightarrow(\mathbf{C}, 0)$ com singularidade isolada, definida como o grau da aplicação $\frac{\partial f}{|a f|}: S_{\varepsilon} \rightarrow S_{1}$. O resultado principal do capítulo é o Teorema 2.5. o qual diz que $\mu_{g}(f)$ é o número de pontos críticos nào degenerados de uma perturbação estável de $f$.

No capítulo 3 introduzimos o conceito de multiplicidade algébrica, $\mu_{a}(f)=\operatorname{dim} \mathcal{O}_{\pi} / J f$, de um gerne holomorfo em 0 . Os resultados principais do capitulo são os Teoremas 3.1 e 3.2 que garantem respectivamente:

$-\mu_{a}(f)<\infty$ se, e somente se. 0 é singularidade isolada ou valor regular de $f$.

$-\mu_{o}(f)<\infty$ entào existe um difeomorfismo biholomorfo $h$ tal que $f \circ h$ é um polinômio.

O capitulo 4 é o capítulo central do trabalho. Neste capitulo, mostramos a equivalência entre a multiplicidade gradiente e o número de Milnor (Teorema 4.1) e a equivalência entre multiplicidade gradiente e a multiplicidade algébrica (Teorema 4.2).

Finalmente, o objetivo do capítulo 5 é apresentar uma fórmula devido à Milnor e Orlik [4], para o cálculo do número de Milnor para polinómios quase homogêneos com singularidade isolada. 


\section{Capítulo 1}

\section{O NÚMERO DE MILNOR}

Seja $f\left(z_{1}, \ldots, \tilde{z}_{n}\right)$ um polinômio nào constante em $n$ variáveis complexas, e seja $\checkmark$ o conjunto algébrico constituido de todas $n$-uplas $z=\left(z_{1}, \ldots, z_{n_{1}}\right)$ de números complexos com $f(z)=0$.

Em [1]. Milnor estudou a topologia de $V$ numa vizinhança de um ponto $z^{\circ}$, utilizando o método de Brauner. que consiste em estudar a interseç̧âo da hipersuperficie $V$ com uma esfera $S_{\varepsilon}$ de raio $\varepsilon$ suficientemente pequeno.

Para descrever a topologia de $\Lambda^{\prime}=V \cap S_{\varepsilon}$. Milnor considerou a fibração $\phi: S_{\varepsilon}-h \rightarrow S^{1}$, onde $\phi(z)=\frac{f(z)}{|f(z)|}$.

No parágrafo 1.1. enunciamos os resultados básicos do trabalho de Milnor, que sảo o Teorema da Fibraçâo e os teoremas sobre a topologia da fibra. No parágrafo 1.2. demonstramos que cada fibra tem o mesmo tipo de homotopia de un bouquet de esferas. O número de Milnor é definido como o número de esferas do bouquet. No parágrafo 1.3 temos como resultado principal o Teorema de Lê Dûng Tráng que mostra que o número de Milnor é um invariante do tipo topológico de $V=f^{-1}(0)$.

\subsection{O Teorema da Fibração e a Topologia da Fibra}

Seja $f:\left(\mathbf{C}^{n}, 0\right) \rightarrow(\mathbf{C}, 0)$ um polinômio não constante em $n$ variáveis, e $V=f^{-1}(0)$ a hipersuperfície complexa definida por $f$. 
Definição $1.1 w \in \mathrm{C}^{n} \dot{\epsilon}$ ponto crítico de $f$ se $d f(w)=0$ e é ponto crítico isolado se existe vizinhança $U$ de $w \in m \mathbf{C}^{n}$ tal que $d f(z)=0, z \in U$ implica $z=w$.

Definição 1.2 Seja $h: \mathbf{C}^{n} \rightarrow \mathbf{C}$ uma função holomorfa. Definimos o gradiente $d \epsilon h, \operatorname{grad} h(z)$, por

$$
\operatorname{grad} h=\left(\frac{\overline{\partial h}}{\partial z_{1}}, \ldots, \frac{\partial \bar{h}}{\partial \bar{z}_{n}}\right)
$$

Tal definição é motivada, para que a derivada de $h$ ao longo de um caminho $z=p(t)$ seja dada por:

$$
\frac{d h}{d t}(p(t))=<\frac{d p}{d t}, \operatorname{grad} h>
$$

onde $\langle u, v\rangle=\sum u_{j} \bar{v}_{j}$ é o produto hermitiano usual.

Dado $\varepsilon>0$, consideremos

$$
\begin{gathered}
D_{\varepsilon}=\left\{z \in \mathbf{C}^{n}:\left|z-z^{o}\right| \leq \varepsilon\right\} \\
S_{\varepsilon}=\partial D_{\varepsilon}=\left\{z \in \mathbf{C}^{n}:\left|z-z^{o}\right|=\varepsilon\right\} \\
K=V \cap S_{\varepsilon}
\end{gathered}
$$

O teorema a seguir estabelece uma relação entre a topologia de $K$ e a de $I^{\circ}$. A demonstraçào pode ser encontrada em [1] e [5].

Teorema 1.1 Para $\varepsilon>0$ suficientemente pequeno $V \cap D_{\varepsilon}$ é homeomorfo ao cone $C\left(K^{\prime}\right)=\left\{t=: 0 \leq t \leq 1, z \in K^{\prime}\right\}$. De fato, o par $\left(D_{\varepsilon}, V \cap D_{\varepsilon}\right) \epsilon_{\text {t homeomorfo ao }}$ $\operatorname{par}\left(C\left(S_{\varepsilon}\right), C\left(K^{\prime}\right)\right)$.

Seja $\phi: S_{\varepsilon}-K \rightarrow S^{1}$ dada por

$$
\phi(z)=\frac{f(z)}{|f(z)|}, \operatorname{com} \phi^{-1}\left(\mathrm{e}^{i \theta}\right)=F_{\theta}
$$

O resultado básico da teoria de Milnor é o teorema da fibração, que mostra que $\phi$ define uma fibração de $S_{\varepsilon}-K$ sobre $S^{1}$ com fibra $F_{\theta}$.

Antes de enunciar este teorema, enunciaremos resultados sobre o conjunto dos pontos críticos de $\phi$ que serão utilizados no decorrer desta dissertação. As demonstrações podem ser encontradas em [1] e [5]. 
Lema $1.1 O$ conjunto dos pontos críticos de $\phi \dot{\epsilon}$ dado por

$$
\left\{z \in S_{\epsilon}: \operatorname{igrad} \log (f(z))=c z, c \in \mathbf{R}\right\}
$$

Lema 1.2 Existe $\varepsilon_{0}$ (raio de Milnor) tal que para todo $z \in C^{n}-V$, com $|z| \leq \varepsilon_{0}$ temos:

1) $z$ e grad $\log (f(z))$ sào lincarmente independentes sobre $\mathrm{C}$ ou

2) $\operatorname{grad} \log (f(z))=\lambda z, \lambda \neq 0 \in \arg (\lambda)<\pi / 4$.

Teorema 1.2 (Fibraçâo de Milnor) $S_{\epsilon} z^{\circ} \dot{\epsilon}$ um ponto qualquer de $V$ e st $S_{\varepsilon} \dot{\epsilon}$ uma esfera suficientemente pequena centrada $\epsilon m z^{\circ}$, entâo $S_{\varepsilon}-K$ é um fibrado diferenciável localmente trivial sobre $S^{1}$, com projeçào $\phi(z)=f(z) /\|f(z)\|$ e fibra $F_{\theta}=\phi^{-1}\left(\mathrm{e}^{i \theta}\right)$

Como o estudo deste trabalho se restringe a germes de hipersuperfícies com singularidades isoladas, ao invés de demonstrarmos o Teorema 1.2, demonstraremos diretamente que $f: D_{\varepsilon} \cap f^{-1}\left(B_{\eta}-\left\{f\left(z_{0}\right)\right\}\right) \rightarrow B_{\eta}-\left\{f\left(z_{0}\right)\right\}$ é a projeção de uma fibraşão localmente trivial, onde $B_{\eta_{l}}=\left\{u \in \mathrm{C}:\left|f\left(z_{0}\right)-u\right| \leq \eta\right\}$.

Teorema 1.3 Suponhamos $z_{0}=0$ singularidade isolada dt $f$. então

$$
f: D_{\varepsilon} \cap f^{-1}\left(B_{\pi_{i}}-\{0\}\right) \rightarrow B_{\eta_{i}}-\{0\}
$$

é a projeção de uma fibraşào localmente trivial.

\section{Demonstração:}

O resultado seguirá do Teorema de Ehresmann [5] se as seguintes hipóteses forem verificadas:

(i) $f$ é própria;

(ii) as variedades $D_{\varepsilon} \cap f^{-1}\left(B_{\eta}-\{0\}\right)$ e $B_{\eta}-\{0\}$ sào conexas:

(iii) $f$ e $\left.f\right|_{a\left(D_{c} \cap f^{-2}\left(B_{\eta}-\{0\}\right)\right)}$ sào submersòes;

De fato, temos:

(i) Seja $z_{n}$ sequência em $D_{\varepsilon} \cap f^{-1}\left(B_{\eta}-\{0\}\right)$ sem subsequência convergente. Como $z_{n} \in D_{\varepsilon}$ temos que existe subsequência $z_{n_{k}}$ em $D_{\varepsilon}$ tal que $z_{n_{k}} \rightarrow v \in V$, logo $f\left(z_{n_{k}}\right) \rightarrow f(v)=0$, por continuidade da $f$. Portanto $f\left(z_{n}\right)$ não tem subsequência convergente. Logo, $f$ é própria. 
(ii) Para mostrar que $D_{\varepsilon} \cap f^{-1}\left(B_{\eta}-\{0\}\right)$ é variedade diferenciável conexa, basta observar que qualquer caminho ligando pontos $P$ e $Q$ de

$D_{\varepsilon} \cap f^{-1}\left(B_{\eta}-\{0\}\right)$ pode ser aproximado por um caminho transversal à $I^{\prime}$ e que, portanto, nào encontra $V$.

(iii) Temos que $f$ é uma submersào em $D_{\varepsilon} \cap f^{-1}\left(B_{\eta}-\{0\}\right)$ já que 0 é singularidade isolada. Mais ainda. $\left.f\right|_{\partial\left(D_{t} \cap f^{-1}\left(B_{\eta}-\{0\}\right)\right)}$ é submersão. De fato, se existisse $z \in S_{\varepsilon}$ tal que $\left\langle v\right.$, grad $f(z)>=0 \forall v \in T_{z} S_{\varepsilon}$ teriamos também $<i v, \operatorname{grad} f(z)>=0, \forall v \in T_{z} S_{\varepsilon}$. Assim, teriamos que $z$ seria singularidade de $f$, o que é um absurdo.

Observação: Os resultados do Teorema 1.2 e do Teorema 1.3 se correspondem, pois se $c \in C$ possui módulo suficientemente pequeno então $f^{-1}(c) \cap D_{\varepsilon}$ é difeomorfo à $F_{\theta}[1]$.

Os result ados sobre a topologia da fibra $F_{\theta}$ sâo descritos a seguir. Os mesmos podem ser encontrados em [1] e [5].

Lema 1.3 Existe uma aplicaçâo diferenciável

$$
s_{\theta}: F_{\dot{\theta}} \rightarrow \mathbf{R}_{+}
$$

tais que seus pontos críticos sâo nâo degenerados, com indice de Morse $\geq n-1$.

Teorema 1.4 Cada fibra $F_{\theta} \in S_{\varepsilon}-h$ tém o mesmo tipo de homotopia que um complexo CW finito, de dimensôes $n-1$ en respectivamente.

Para o caso particular em que $f$ tem singularidade isolada em $z^{\circ}=0$ temos:

Lema 1.4 Para $\varepsilon$ suficientemente pequeno. o fecho $\epsilon m S_{\varepsilon}$ de cada fibra $F_{\theta} \dot{\epsilon}$ uma variedade diferenciárel de dimensāo real $2 n-2$. com bordo. O interior dessa variedade $\epsilon F_{\theta} \in$ o bordo é precisamente $K^{\prime}$.

Teorema $1.5 A$ homologia de $F_{\theta}$ está concentrada nas dimensóes 0 e $n-1$. Além disto, $H_{o}\left(F_{\theta}\right) \simeq \mathbf{Z}$. 


\subsection{Definição do Número de Milnor}

Quando $f$ tem singularidade isolada em 0 . o teorema abaixo caracteriza o tipo de homotopia da fibra $F_{\theta}$

Teorema 1.6 Cada fibra $F_{\theta}$ tem o mesmo tipo de homotopia de um bouquet. $S^{n-1}$ r.... $S^{n-1} d \epsilon$ esferas.

Os lemas 1.5 e 1.6 abaixo serào necessários para a demonstraçào do Teorema 1.6 .

Lema 1.5 A variedade compacta com bordo. $\bar{F}_{\theta}$. está mergulhada $\epsilon m S_{\varepsilon} d \epsilon$ modo que tem o mesmo tipo de homotopia que seu complementar $S_{\varepsilon}-\bar{F}_{\theta}$.

\section{Demonstração:}

Como $\bar{F}_{\theta}$ é uma subvariedade de $S_{\varepsilon}$. a inclusão fornece um mergulho. Agora, o Teorema da Fibraçào fornece a fibraçào localmente trivial $\phi: S_{\varepsilon}-K^{r} \rightarrow S^{1}$. Segue-se que a restrição

$$
0: S_{\varepsilon}-\left(K \cup \sigma^{-1}\left(\mathrm{e}^{i \theta}\right)\right) \rightarrow S^{1}-\left\{\mathrm{e}^{i \theta}\right\} .
$$

é uma fibraçào localmente trivial. Mas. $S^{1}-\left\{e^{i t}\right\}$ é contrátil e, portanto, a fibraçào é globalmente trivial. ou seja. $S_{\varepsilon}-\bar{F}_{\dot{\theta}}=S_{\varepsilon}-\left(K \cup F_{\theta}\right)$ (Lema 1.4) é difeomorfa à $\left(S^{1}-\left\{\mathrm{e}^{i \theta}\right\}\right) \times F_{\bar{\theta}}$ onde $F_{\bar{\theta}}$ é qualquer fibra diferente de $F_{6}$. Usando novamente a contratibilidade de $S^{1}-\left\{e^{i \theta}\right\}$. temos que $S_{\varepsilon}-\bar{F}_{\theta}$ tem $F_{\bar{\theta}}$ como um retrato por deformação. Como as fibras sào difeomorfas. temos que $S_{\varepsilon}-\bar{F}_{\theta}$ tem o mesmo tipo de homotopia que $F_{\theta}$. Além disso, $\bar{F}_{\theta}$ e $F_{\theta}$ tem o mesmo tipo de homotopia, e: portanto segue o resultado.

Lema 1.6 A fibra $F_{\theta} \dot{\epsilon}^{\prime}$ simplesmente conexa para $n \geq 3$.

\section{Demonstração:}

Consideremos a funçào $-s_{\theta}: \bar{F}_{\theta} \rightarrow \mathbf{R}_{+}$, onde $s_{\theta}$ é a função de Morse obtida pelo Lema 1.3. Então, $-s_{\theta}$ é uma função de Morse em $\bar{F}_{\theta}$ cujos pontos críticos 
têm índice $i \leq n-1$. Observando que o índice de $-s_{\theta}$ associado ao menor valor crítico é zero, concluímos que $\bar{F}_{\theta}$ é obtido de um disco $D^{2 n-2}$ pela colagem de células de dimensâo menor ou igual a $n-1$. Temos que $S_{\varepsilon}-D^{2 n-2}$ é contrátil e, portanto, simplesmente conexo. Se colarmos uma célula $\epsilon_{\lambda}$ de dimensâo $\lambda \leq n-1$ a $D^{2 n-2}$, o grupo fundamental de $S_{\varepsilon}-\left(D^{2 n-2} \cup \epsilon_{\lambda}\right)$ nào se altera desde que $\lambda \leq \operatorname{dim} S_{\varepsilon}-3=2 n-4$. De fato. dados dois caminhos em $S_{\varepsilon}$. podemos tomar uma homotopia $F$ entre eles, tal que $F$ seja transversal à $\epsilon_{\lambda}$, o que, nestas dimensòes implica em $F$ nào encontrar $\epsilon_{\lambda}$.

Portanto para $n \geq 3$ e $\lambda \leq n-1 \leq 2 n-4$, temos

$$
\pi_{1}\left(S_{\varepsilon}-\left(D^{2 r-2} \cup \epsilon_{\lambda}\right)\right) \simeq \pi_{1}\left(S_{\varepsilon}-D^{2 n-2}\right)=0
$$

Mas, $\bar{F}_{\theta}$ é obtido de $D^{2 n-2}$ após a colagem de um número finito de células. Portanto.

$$
0=\pi_{1}\left(S_{\varepsilon}-D^{2 n-2}\right) \simeq \pi_{1}\left(S_{\varepsilon}-\bar{F}_{\theta}\right) .
$$

Usando o Lema 1.5 obtemos:

$$
0=\pi_{1}\left(\bar{F}_{6}\right) \simeq \pi_{1}\left(S_{\varepsilon}-\bar{F}_{\theta}\right) \simeq \pi_{1}\left(F_{\theta}\right) .
$$

Portanto, $F_{\theta}$ é simplesmente conexa.

Observação: L'sando o Teorema 1.4 e o Teorema de Hurtuicz $[8,7.5 .5]$ : "Se $X$ é simplesmente conexo e existe $q \geq 2$ tal que $H_{i}(X)=0$ para $i<q$, então $\pi_{i}(X)=0$ para $i<$ g e existe um isomorfismo $\pi_{q}(X) \simeq H_{q}(X)$, "concluímos que $F_{\theta}$ é $(n-2)$-conexa.

Passemos a demonstração do Teorema 1.6.

Afirmamos que $H_{n-1}\left(F_{\theta}\right)$ é abeliano livre. De fato. se $H_{n-1}\left(F_{\theta}\right)$ possuísse parte de torçâo. teríamos cohomologia nâo trivial em dimensâo $n$ (sendo $H_{*}\left(F_{\theta}\right)$ finitamente gerado, $H^{n}\left(F_{\theta}\right)=F\left(H_{n}\left(F_{\theta}\right)\right)+T\left(H_{n-1}\left(F_{\theta}\right)\right)$ onde $F$ é a parte livre e $T$ é a parte de torçâo de $\left.H_{*}\left(F_{\theta}\right)\right)$, o que é impossível pelo Teorema 1.4.

Se $n \geq 3$, usando o Teorema de Hurewicz, temos que

$$
\pi_{n-1}\left(F_{\theta}\right) \simeq H_{n-1}\left(F_{\theta}\right) \text {. }
$$


Seja $\left(S^{n-1} v \ldots v S^{n-1}, p_{0}\right) \rightarrow\left(F_{\theta}, p_{1}\right)$ uma aplicaçào continua que leva o ponto base $p_{o}$ no ponto base $p_{1}$ e que leva as esferas $\left(S^{n-1}, p_{o}\right)$ do bouquet nos geradores de $\pi_{n-1}\left(F_{\theta}, p_{1}\right)$. Uma tal aplicaçào induz um isomorfismo entre as homologias

$$
H_{n-1}\left(S^{n-1} \vee \ldots \vee S^{n-1}\right) \simeq H_{n-1}\left(F_{\theta}\right)
$$

Como $F_{\theta}$ e $S^{n-1}$ v...v $S^{n-1}$ são espaços $C W^{\prime}$ simplesmente conexos, a aplicação acima entre estes espaços, é de fato, uma equivalência de homotopia ( Ttortma d€ Whitehtad, [8, 7.6.25].

Se $n=2$ temos o caso clássico. Neste caso. $\bar{F}_{\theta}$ é uma superfície conexa compacta orientável e com bordo. Logo, pela classificaçâo dessas variedades de dimensão 2 , segue-se que $\bar{F}_{\theta}$ tem o mesmo tipo de homotopia que um bouquet de esferas $S^{1}$.

Definição 1.3 O número de esferas $S^{n-1}$ do bouquet, ou o número de geradorts da homologia média da fibra $F_{\theta}$ é chamado número de Milnor de $f \in m 0 \epsilon \epsilon$ denotado por $\mu$.

Observação: Para $c$ suficientemente pequeno temos que $F_{c}=f^{-1}(c)$ é difeomorfo a $F_{\theta}$. Portanto cada uma dessas esferas representa um gerador de $H_{r_{-1}}\left(F_{c}\right)$. Estas esferas foram chamadas por Lefschet $z$ de "ciclos evanescentes". Uma explicação para essa terminologia é que quando $c \rightarrow 0, F_{c} \rightarrow V=f^{-1}(0)$. que é contrátil, por ser homeomorfo a um cone (Teorema 1.1).

Exemplo 1.1 Seja $f:\left(\mathbf{C}^{n}, 0\right) \rightarrow(\mathbf{C}, 0)$ dada por $f\left(z_{1}, \ldots z_{n}\right)=z_{1}^{2}+\ldots+z_{n}^{2}$.

Escreva $z_{j}=x_{j}+i y_{j}$. Sejam

$$
\begin{gathered}
B_{\eta_{1}}=\{w \in \mathrm{C}:|w| \leq \eta\}, B_{\eta}^{*}=B_{\eta}-\{0\} \\
D_{\varepsilon}=\left\{z \in \mathbf{C}^{\eta}:|z| \leq \varepsilon\right\} \\
Y=D_{\varepsilon} \cap f^{-1}\left(B_{\eta}\right) . \quad \hat{Y}=D_{\varepsilon} \cap f^{-1}\left(B_{\eta}^{*}\right)
\end{gathered}
$$

Pelo Teorema 1.3, sabemos que $f: Y \rightarrow B_{\eta}^{*}$ é uma fibraçào $C^{\infty}$ localmente trivial, cuja fibra típica $Y_{w}\left(w \in B_{\eta}^{*}\right)$ é difeomorfa à fibra de Milnor $F_{\theta}$ associada a $f$.

Vemos que $\tilde{Y}$ e $Y_{\eta}$ são descritos por:

$$
\tilde{Y}=\left\{z \in \mathbf{C}^{n}:\left|z_{1}\right|^{2}+\ldots+\left|z_{n}\right|^{2} \leq \varepsilon^{2} \mathrm{e}\left|z_{1}^{2}+\ldots+z_{n}^{2}\right| \leq \eta\right\}
$$




$$
Y_{\eta}=\left\{z \in Y: z_{1}^{2}+\ldots+z_{n}^{2}=\eta\right\}=f^{-1}(\eta) \cap Y
$$

Em coordenadas reais, $Y_{\eta}$ é dado por:

$$
\sum x_{j}^{2}+\sum y_{j}^{2} \leq \varepsilon^{2}, \quad \sum x_{j}^{2}-\sum y_{j}^{2}=\eta \text { e } \sum x_{j} y_{j}=0
$$

Segue que $\sum y_{j}^{2} \leq \frac{\varepsilon^{2}-\eta}{2}$. Como $\sum x_{k}^{2} \neq 0$, sejam

$$
u_{j}=\frac{x_{j}}{\sqrt{\sum x_{k}^{2}}} \text { e } v_{j}=\frac{y_{j}}{\sqrt{\frac{\varepsilon^{2}-I_{j}}{2}}}
$$

Obtemos então um difeomorfismo entre $Y_{\eta}$ e o conjunto definido por:

$$
\sum u_{j}^{2}=1 \cdot \sum v_{j}^{2} \leq 1 \text { e } \sum u_{j} v_{j}=0
$$

Geometricamente isto significa considerar a esfera $S^{n-1}=\left\{\sum u_{j}^{2}=1\right\}$ em $\mathbf{R}^{n}$ e tomar os vetores $\left(v_{1}, \ldots, v_{n}\right)$ tangentes a $S^{n-1}$ no ponto $\left(u_{1}, \ldots, u_{n}\right)$ que tem comprimento menor ou igual a 1 . En outras palavras. $Y_{\eta_{1}}$ é difeomorfa ao fibrado por bolas unitárias a $S^{n-1}$ e o bordo $\partial \xi_{r_{1}} \simeq K$ é difeomorfo ao fibrado esférico unitário tangente a $S^{n-1}$. Estes sào denotados por $T S_{|| \leq 1}^{n-1}$ e $T S_{||=1}^{n-1}$ respectivamente.

Como $T S_{|| \leq 1}^{n-1}$ se retraj a $S^{r_{-1}}$ temos $H_{0}\left(Y_{\eta_{l}}\right) \simeq \mathrm{Z} . H_{n_{-1}}\left(Y_{\eta_{1}}\right) \simeq \mathrm{Z}$ e um gerador de $H_{n-1}\left(Y_{\eta}\right)$ é representado pela esfera $S^{n-1}$.

Portanto, o número de Milnor de $f$ é 1 .

\subsection{O Número de Milnor como Invariante To- pológico}

Definição 1.4 Duas funçòts holomorfas $f \in g:\left(\mathrm{C}^{n}, 0\right) \rightarrow(\mathrm{C} .0)$ com 0 ponto

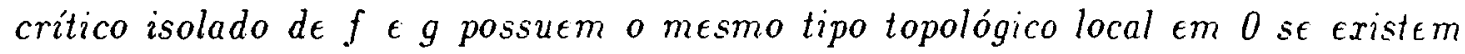
vizinhanças $U \in V$ de 0 em $\mathbf{C}^{n}$ e um homeomorfismo $\psi:(U, 0) \rightarrow(V, 0)$ tais que $\psi\left(f^{-1}(0) \cap U\right)=g^{-1}(0) \cap V$.

$\mathrm{O}$ seguinte teorema devido a $L \hat{e}$ Dúng Tráng, mostra que o número de Milnor é um invariante completo do tipo topológico de $V$. 
Teorema 1.7 (Lí Düng Tráng [17]) Se dois germes f e g possucm o mesmo tipo topológico entào $\mu(f)=\mu(g)$.

A demonstração deste teorema depende do seguinte lema:

Lema 1.7 Existe $\varepsilon_{0}>0$ tal que para tados $\varepsilon \epsilon \varepsilon^{\prime} \operatorname{com} 0<\varepsilon<\varepsilon_{o} \epsilon 0<\varepsilon^{\prime}<\varepsilon_{0}$; $D_{\epsilon}-f^{-1}(0) \in D_{\varepsilon^{\prime}}-g^{-1}(0)$ sâo homotopicamente equiralentes.

\section{Demonstração:}

Por hipótese existe um homeomorfismo $\psi^{\prime}:(l: 0) \rightarrow(V, 0)$ tais que $\psi\left(f^{-1}(0) \cap U\right)=g^{-1}(0) \cap V$.

Seja $\varepsilon_{0}$ tal que as propriedades do Teorema 1.1 se verificam tanto para $f$ como para $g$. ou seja. para $0<\varepsilon<\varepsilon_{0}$

$$
\begin{aligned}
& \left(D_{\varepsilon} \cdot f^{-1}(0) \cap D_{\varepsilon}\right) \simeq\left(C\left(S_{\varepsilon}\right), C\left(K_{f}\right)\right) \\
& \left(D_{\varepsilon} \cdot g^{-1}(0) \cap D_{\varepsilon}\right) \simeq\left(C\left(S_{\varepsilon}\right), C\left(K_{g}\right)\right)
\end{aligned}
$$

Portanto, temos:

$$
\begin{aligned}
& D_{\varepsilon}-f^{-1}(0) \sim S_{\varepsilon}-K_{f} \\
& D_{\varepsilon}-g^{-1}(0) \sim S_{\varepsilon}-K_{g}
\end{aligned}
$$

onde " $\sim$ "indica mesmo tipo de homotopia.

Sejam $\varepsilon_{1}$. $\varepsilon_{2}$. $\varepsilon_{3}$. $\varepsilon_{4}$ tais que $0<\varepsilon_{1}<\varepsilon_{2}<\varepsilon_{0} .0<\varepsilon_{3}<\varepsilon_{4}<\varepsilon_{0}$ e $\psi\left(D_{\varepsilon_{1}}\right) \subset D_{\varepsilon_{3}} \subset \varepsilon^{\prime}\left(D_{\varepsilon_{2}}\right) \subset D_{\varepsilon_{4}}$.

Tomando a sequência de homotopia

$$
\begin{aligned}
\pi_{i}\left(\psi\left(D_{\varepsilon_{1}}\right)-g^{-1}(0) . x\right) \rightarrow & \pi_{i}\left(D_{\varepsilon_{3}}-g^{-1}(0), x\right) \rightarrow \pi_{i}\left(\imath \cdot\left(D_{\varepsilon_{2}}\right)-g^{-1}(0) . x\right) \rightarrow \\
& \rightarrow \pi_{i}\left(D_{\varepsilon_{4}}-g^{-1}(0) . x\right)
\end{aligned}
$$

com $x \in \psi\left(D_{\varepsilon_{1}}\right)-g^{-1}(0)$ e observando ainda que.

$$
\begin{gathered}
\pi_{i}\left(D_{\varepsilon}-f^{-1}(0), y\right) \simeq \pi_{i}\left(\psi\left(D_{\varepsilon}\right)-g^{-1}(0), \psi(y)\right) \\
\pi_{i}\left(D_{\varepsilon_{1}}-f^{-1}(0), y\right) \simeq \pi_{i}\left(D_{\varepsilon_{2}}-f^{-1}(0), y\right) \\
\pi_{i}\left(D_{\varepsilon_{3}}-g^{-1}(0), x\right) \simeq \pi_{i}\left(D_{\varepsilon_{1}}-g^{-1}(0), x\right)
\end{gathered}
$$


concluimos que $\pi_{i}\left(D_{\varepsilon}-f^{-1}(0), y\right) \simeq \pi_{i}\left(D_{\varepsilon^{\prime}}-g^{-1}(0)\right.$. $\left.x\right)$ para todos $\varepsilon$ e $\varepsilon^{\prime}$ com $0<\varepsilon<\varepsilon_{o}$ e $0<\varepsilon^{\prime}<\varepsilon_{o}$.

Como $D_{\varepsilon}-f^{-1}(0)$ e $D_{\varepsilon^{\prime}}-g^{-1}(0)$ possuem tipo topológico de um complexo $C W$ finito obtemos uma equivalència homotópica entre $D_{\varepsilon}-f^{-1}(0)$ e $D_{\varepsilon^{\prime}}-g^{-1}(0)$ ([8, p. 406]).

\section{Demonstração do Teorema 1.7}

Sejam:

$$
\begin{gathered}
o_{f}: S_{\varepsilon}-h_{f} \rightarrow S^{1} \mathrm{e} \\
o_{g}: S_{\varepsilon^{\prime}}-h_{g} \rightarrow S^{1}
\end{gathered}
$$

as fibraçôes de Milnor de $f$ e $g$ : com fibras $F_{\theta}$ e $F_{\theta}^{\prime}$ respectivamente.

Consideremos o diagrama

$$
\begin{array}{ccc}
\boldsymbol{X}_{f} & \longrightarrow & \mathbf{R} \\
\downarrow \downarrow & \downarrow \in x p \\
S_{\varepsilon}-H_{f} & \stackrel{\phi_{f}}{\longrightarrow} & S^{1}
\end{array}
$$

onde $X_{f}$ é o pull-back de $\mathbf{R}$ pela $\phi_{f}$, ou seja.

$$
X_{f}=\left\{(x . y): o_{f}(x)=\epsilon x p(y)\right\} \subset\left(S_{\varepsilon}-h_{f}^{\prime}\right) \times \mathbf{R}
$$

e. portanto um recobrimento de $S_{\varepsilon}-K_{f}$ associado a $\left(\varphi_{f}\right)_{*}^{-1}(0)=\operatorname{ker}\left(\phi_{f}\right) *$ com

$$
\left(\phi_{f}\right): \pi_{1}\left(S_{\varepsilon}-K_{f}, x\right) \longrightarrow \pi_{1}\left(S^{1}, \phi_{f}(x)\right)
$$

Além disso, como $\operatorname{ker}\left(\phi_{f}\right)_{\text {. }}$ é um subgrupo normal temos

$$
\operatorname{Aut}\left(X_{f}\right) \simeq \frac{\pi_{i}\left(S_{\varepsilon}-K_{f}, x\right)}{\operatorname{ker}\left(\phi_{f}\right)_{*}} \simeq \mathbf{Z}
$$


onde $A$ ut $\left(X_{f}\right)$ é o grupo dos automorfismos de $X_{f}$, ou seja, $X_{f}$ é um recobrimento cíclico infinito de $S_{\varepsilon}-K_{j}[10$. p. 159].

Por outro lado também podemos obter $X_{f}$ como o pull-back de $S_{\varepsilon}-K_{f}$ pela $\epsilon x p$ :

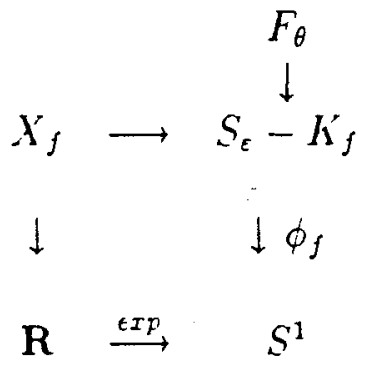

Entào $\mathrm{I}_{f} \rightarrow \mathbf{R}$ é uma fibraçào com fibra $F_{\theta}$. Como a base é contrátil, entào $X_{f} \simeq F_{\theta} \times \mathbf{R}$.

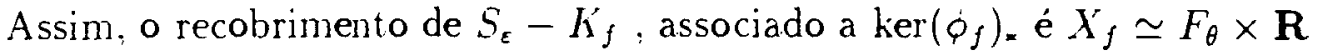

Consideremos agora o recobrimento ciclico infinito:

$$
p_{f}: Y_{f} \longrightarrow D_{\varepsilon}-f^{-1}(0)
$$

de $D_{\varepsilon}-f^{-1}(0)$. associado a imagem isomorfa de $\operatorname{ker}\left(o_{f}\right)=$ : já que

$$
\pi_{1}\left(D_{\varepsilon}-f^{-1}(0) . x\right) \simeq \pi_{1}\left(S_{\varepsilon}-h_{f}, x\right) \stackrel{\left(\phi_{f}\right)}{\longrightarrow} \pi_{1}\left(S^{1} \cdot \phi_{f}(x)\right)
$$

onde $x \in S_{\varepsilon}-K_{f}$

Assim temos

$$
\begin{array}{ccc}
X_{f} & \hookrightarrow & Y_{f} \\
p \downarrow & & \downarrow p_{f} \\
S_{\varepsilon}-K_{f} & \hookrightarrow & D_{\varepsilon}-f^{-1}(0)
\end{array}
$$

Como a inclusâo de $S_{\varepsilon}-K_{f}$ é uma equivalência de homotopia, segue-se que a aplicação entre os recobrimentos $X_{f}$ e $Y_{f}$ define também uma equivalência de homotopia entre estes espaços. 
Seja $p_{g}: Y_{g} \rightarrow D_{\varepsilon}-g^{-1}(0)$ o recobrimento cíclico infinito de $D_{\varepsilon}-g^{-1}(0)$, definido analogamente.

Consideremos $\varepsilon^{\prime}>0$ tal que $\iota^{\prime}\left(D_{\varepsilon^{\prime}}\right) \subset D_{\ell}$. Entào temos o seguinte diagrama

$$
\begin{array}{ccccc}
F_{\theta} \times \mathrm{R} \sim \mathrm{Y}_{f} & \longrightarrow & Z_{g} & \hookrightarrow & Y_{g} \sim F_{\theta}^{\prime} \times \mathbf{R} \\
p_{f} \downarrow & & \overline{p_{g}} \downarrow & & \downarrow p_{g} \\
D_{\varepsilon^{\prime}}-f^{-1}(0) & \stackrel{\longrightarrow}{\longrightarrow} & \psi^{\prime}\left(D_{\varepsilon^{\prime}}\right)-g^{-1}(0) & \stackrel{i}{\hookrightarrow} & D_{\varepsilon}-g^{-1}(0)
\end{array}
$$

onde a inclusão $i: \psi\left(D_{\varepsilon^{\prime}}\right)-g^{-1}(0) \hookrightarrow D_{\varepsilon}-g^{-1}(0)$ é equivalência de homotopia (pelo lema anterior) e $Z_{g}=p_{g}^{-1}\left(\psi^{\prime}\left(D_{\varepsilon^{\prime}}\right)-g^{-1}(0)\right)$ é o recobrimento cíclico infinito de $\zeta^{\prime}\left(D_{\varepsilon^{\prime}}\right)-g^{-1}(0)$. pull-back de $Y_{g}$.

Portanto $Z_{g} \hookrightarrow Y_{g}$ é uma equivalência de homotopia.

Pela unicidade do recobrimento cíclico infinito de $D_{\varepsilon^{\prime}}-f^{-1}(0)$. concluímos que os recobrimentos $Y_{f}$ e $Z_{g}$ são isomorfos.

Portanto, $Y_{f} \sim Y_{g}$ e como consequência, $F_{\theta} \sim F_{\theta}^{\prime}$. Logo $\left.\left.\mu_{(} f\right)=\mu_{(} g\right)$.

Observação 1: Para $n>2$ é possivel simplificar o argumento acina usando a sequência de homotopia da fibração.

De fato. temos que $F_{\theta}$ e $F_{\theta}^{\prime}$ são $(n-2)$-conexas (ver Obsevação da p. 8). Portanto. tomando a sequência exata dada pela fibraçào, ver [15, p. 90], vemos:

$$
0 \rightarrow \pi_{n-1}\left(F_{\theta}\right) \rightarrow \pi_{n-1}\left(S_{\varepsilon}-f^{-1}(0)\right) \rightarrow 0
$$

Logo, $\pi_{n-1}\left(F_{\theta}\right) \simeq \pi_{n-1}\left(S_{\varepsilon}-f^{-1}(0)\right) \simeq \pi_{n-1}\left(D_{\varepsilon}-f^{-1}(0)\right)$

Analogamente $\pi_{n-1}\left(F_{\theta}^{\prime}\right) \simeq \pi_{n-1}\left(S_{\varepsilon^{\prime}}-g^{-1}(0)\right) \simeq \pi_{n-1}\left(D_{\varepsilon^{\prime}}-g^{-1}(0)\right)$.

Portanto, $\pi_{n-1}\left(F_{\theta}\right) \simeq \pi_{n-1}\left(F_{\theta}^{\prime}\right)$, pelo Teorema de Hurewicz (ver Observaçào da p. 8)

$$
H_{n-1}\left(F_{\theta}\right) \simeq \pi_{n-1}\left(F_{\theta}\right) \simeq \pi_{n-1}\left(F_{\theta}^{\prime}\right) \simeq H_{n-1}\left(F_{\theta}^{\prime}\right)
$$

Portanto $\mu(f)=\mu(g)$. 
Observação 2: $\mu(f)=\mu(g)$ não implica que $f$ e $g$ possuem o mesmo tipo topológico.

Por exemplo, sejam

$$
f, g:\left(\mathbf{C}^{2}, 0\right) \rightarrow(\mathbf{C}, 0)
$$

tais que

$$
f\left(z, u^{\prime}\right)=z^{3}+w^{3} \text { e } g(z, w)=z^{2}+u^{5}
$$

Com o resultado a ser visto no capítulo 5 , pode-se verificar facilmente que $\mu(f)=\mu(g)=4$.

Mas $g^{-1}(0)$ é um cone sobre o nó $K^{\prime}$ do tipo $(2,5)([1])$ e $f^{-1}(0)$ é formado por três retas complexas que interceptam na origem.

No entanto, se uma deformaçào $f_{t}$ de um germe $f$ tem número de Milnor $\mu\left(f_{t}\right)$ constante, as variedades $V_{t}=f_{t}^{-1}(0)$ sào topologicamente equiralentes.

Este resultado foi provado por Lê Dùng Tráng e Ramanujam para famílias a um parâmetro ([18]) e estendida para famílias a vários parâmetros por Timourian em ([19]). 


\section{Capítulo 2}

\section{Caracterização Geométrica do Número de Milnor}

Neste capítulo vamos introduzir o conceito de multiplicidade gradiente. $\mu_{g}(f)$, associada a um germe $f:\left(\mathbf{C}^{n}, 0\right) \rightarrow(\mathbf{C}, 0)$, com singularidade isolada.

Este invariante da singularidade será caracterizado (Teorema 2.4) como número de soluçōes da equaçào $\partial f(z)-a=0$, para um valor regular $a$ suficientemente próximo de 0 .

Como consequência deste result ado será possivel mostrar que $\mu_{g}(f)$ é o número de pontos críticos nào degenerados de uma perturbaçào estável de $f$.

\subsection{O Grau de uma Aplicação}

Sejam $X, Y$ e $Z$ variedades diferenciáveis orientadas e sem bordo. com $X$ compacta. $Z$ subvariedade fechada de $Y$, e $\operatorname{dim} X+\operatorname{dim} Z=\operatorname{dim} Y$.

Seja $h: X \rightarrow Y$. Pelo Teorema de Transversalidade [7]. podemos supor $h$ transversal a $Z$. Assim. $h^{-1}(Z)$ tem um número finito de pontos.

Para cada $x \in h^{-1}(Z)$, definimos o sinal de $d h_{x}, \operatorname{sinal}\left(d h_{x}\right)$, por +1 ou -1 conforme $d h_{x}$ preserve ou inverta orientação.

Definição 2.1 O número de intersecçâo $I(h, Z)$ é a soma dos sinais de dh $h_{x}$, para $x \in h^{-1}(Z)$, isto é, 


$$
I(h, Z)=\sum_{x \in h^{-1}(Z)} \operatorname{sinal}\left(d h_{x}\right)
$$

Quando $X$ também é uma subvariedade de $Y$, o número de interseç̧ào de $X \operatorname{com} Z, I(X, Z)$, é definido como sendo o número de interseçào da aplicação inclusào de $X$ em $Z$.

Suponha que $X$ seja a fronteira de uma variedade compacta $W$ e que $h$ se estenda a uma aplicaşào $H: W \rightarrow Y$. Podemos supor $H$ transversal à $Z$ (Teorema da Extensão, [9]). Entào $H^{-1}(Z)$ é uma variedade compacta orientada unidimensional com fronteira $\partial H^{-1}(Z)=h^{-1}(Z)$. Pelo Teorema de classificaçào de variedade compacta unidimensional, temos que $\partial H^{-1}(Z)$ tem um número par de pontos. Consequentemente, a soma dos sinais de $d h_{x}, x \in h^{-1}(Z)$ deve ser 0 . Assim, provamos o seguinte:

Lema 2.1 Seja $X$ o bordo de uma variedade compacta $W, X$ orientada como bordo de W. Se $h: X \rightarrow Y$ se estende a uma aplicaşão $H: W \rightarrow Y$ entáo $I(h, Z)=0$.

Em particular, suponhamos que $f_{0}$ e $f_{1}$ sejam homotópicas e ambas transversais à $Z$. Então se $H: J \times X \rightarrow Y$ é uma homotopia entre elas, segue-se que $I(\partial H, Z)=0$, onde $J=[0,1] . \partial H=\left.H\right|_{\partial(J \times X)}, \partial(J \times X)=\{0\} \times X \cup\{1\} \times X$ e

$$
\begin{aligned}
& \left.H\right|_{\{0\} \times X}=f_{0} \\
& \left.H\right|_{\{1\} \times X}=f_{1}
\end{aligned}
$$

Portanto, $0=I(\partial H . Z)=I\left(f_{0} . Z\right)-I\left(f_{1} . Z\right)$

Isto prova un dos teoremas centrais na teoria de intersecçào.

Teorema 2.1 Aplicaçòts homotópicas têm o mesmo número de interseç̧ão.

Quando $Y$ é conexo e tem a mesma dimensào de $X$, definimos o grau de uma aplicaçâo $h: X \rightarrow Y$, como sendo o número de intersecção de $h$ com um valor regular $y$, isto é,

$$
\operatorname{grau}(h)=I(h,\{y\})
$$

Teorema 2.2 O inteiro grau $(h)$, nâo depende da escolha do valor regular.

Demonstração: Veja, por exemplo, [2] 


\subsection{A Multiplicidade Gradiente}

Seja $f:\left(\mathbf{C}^{n}, 0\right) \rightarrow(\mathbf{C}, 0)$ um polinomio com ponto crítico isolado em 0 . Tomemos $S_{\varepsilon}$ de modo que 0 seja o único ponto crítico no disco $D_{\varepsilon}$. Podemos definir a aplicaçào

$$
\frac{\partial f}{|\partial f|}: S_{\varepsilon} \rightarrow S_{1}
$$

por $\frac{\partial f}{|a f|}(z)=\frac{\partial f(z)}{|\partial f(z)|}$ onde

$$
\partial f=\left(\frac{\partial f}{\partial z_{1}}, \ldots, \frac{\partial f}{\partial z_{n}}\right)
$$

e $\left(z_{1}, \ldots, z_{n}\right)$ sâo coordenadas usuais de $\mathbf{C}^{n}$ que deste modo possui uma orientação natural. Se $S_{\varepsilon}$ está orientada como bordo de $D_{\varepsilon}$, podemos definir o grau de $\frac{\partial f}{|\partial f|}$ que denotaremos por $\mu_{g}(f)$

Pelo Lema 2.1, $\mu_{g}(f)$ independe de $S_{\varepsilon}$, isto é, se $S_{\delta}$ é uma esfera nas mesmas condiçōes entào

$$
\left.\mu_{g}(f)\right|_{S_{\mathbf{c}}}=\left.\mu_{g}(f)\right|_{s_{\boldsymbol{k}}}
$$

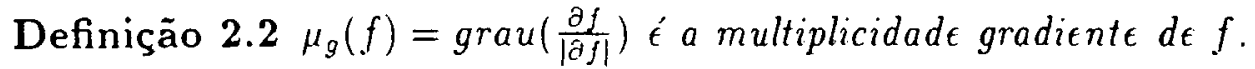

Lema 2.2 (Princípio de Rouché): S€jam $r, L: \mathrm{C}^{n} \rightarrow \mathrm{C}^{n}$ duas aplicaçôes holomorfas $\epsilon$ m uma vizinhanga $d \epsilon z^{\circ}$ tais que $|r(z)|<|L(z)|$ para todo $z \in S_{\varepsilon}$. esfera $d \epsilon$ centro $\epsilon m z^{\circ} \epsilon$ raio z. Então

$$
\operatorname{grau}\left(\frac{L+r}{|L+r|}\right)=\operatorname{grau}\left(\frac{L}{|L|}\right)
$$

ambos restritos a $S_{\varepsilon}$.

\section{Demonstração :}

Pelo Teorema 2.1 basta obter uma homotopia entre estas aplicações. Para isto, é suficiente notar que $|r(z)|<|L(z)|$ implica em $\lambda r(z)+L(z) \neq 0$ para todo $z \in S_{\varepsilon}$ e $0 \leq \lambda \leq 1$. Pois do contrário teríamos

$$
0=|\lambda r(z)+L(z)| \geq-\lambda|r(z)|+|L(z)| \geq-|r(z)|+|L(z)| \Rightarrow|r(z)| \geq|L(z)|,
$$

o que é absurdo. Portanto,

$$
H_{\lambda}(z)=\frac{L(z)+\lambda r(z)}{|L(z)+\lambda r(z)|}
$$


é uma homotopia entre $\frac{L}{|L|}$ e $\frac{L+r}{|L+r|}$, como queriamos.

Como consequência desse lema temos

Teorema $2.3 S \epsilon z^{\circ}=0$ é um ponto crítico nâo degenerado de $f$, isto $\dot{\epsilon}$, a matriz $\left(\frac{\partial^{2} f}{\partial z, \partial z}(0)\right)$ é não singular, entâo $\mu_{g}(f)=1$.

\section{Demonstração :}

Temos $\partial f=L z+r(z)$ onde $L=\frac{\partial^{2} f}{\partial z_{1} \partial z}(0)$ é não singular e $\lim _{|z| \rightarrow 0} \frac{r(z)}{|z|}=0$.

De $\lim _{|=| \rightarrow 0} \frac{r(z)}{|z|}=0$, temos que dado $\delta>0$ e $\lambda=\min _{z \in S_{\delta}}|L(z)|$, então para $\eta=\frac{\lambda}{\delta} \exists \varepsilon, 0<\varepsilon \leq \delta$ tal que se $|z| \leq \varepsilon \Rightarrow|r(z)|<\eta|z| \leq \eta \varepsilon \leq \lambda \leq|L(z)|$.

Portanto, $|r(z)|<|L(z)|, \forall z \in S_{\varepsilon}$. Pelo Lema 2.2 obtemos

$$
\mu_{g}(f)=\left.\operatorname{grau}\left(\frac{\partial f}{|\partial f|}\right)\right|_{S_{e}}=\left.\operatorname{grau}\left(\frac{L+r}{|L+r|}\right)\right|_{S_{\varepsilon}}=\left.\operatorname{grau}\left(\frac{L}{|L|}\right)\right|_{S_{\iota}}
$$

Como $G L(\mathbf{C}, n)$, conjunto das matrízes inversíveis em $\mathbf{C}$, é conexo por caminhos, fazemos uma homotopia $H_{t}$ entre $\frac{L}{|L|}$ e a identidade de modo que

$$
\operatorname{grau}\left(\frac{L}{|L|}\right)=\operatorname{grau}(i d)=1
$$

O objetivo agora, é estabelecer uma relação entre $\mu_{g}$ e os zeros de $\partial f(z)-a$, onde a é valor regular próximo de 0 .

Lema 2.3 Seja $D$ um disco compacto em $\mathbf{C}^{n}$. Se $\partial f^{-1}(0) \cap D \neq \emptyset \epsilon$ $\partial f^{-1}(0) \cap \partial D=\emptyset$ entâo o número de zeros de $\partial f \in m D$ (contados com mul-

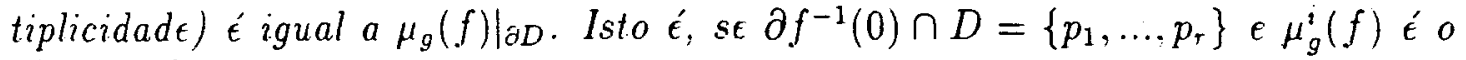
grau de $\frac{\partial f}{|\partial f|}$ restrita a uma pequena esfera em torno de $p_{i}$ então

$$
\left.\mu_{g}(f)\right|_{\partial D}=\sum_{i=1}^{r} \mu_{g}^{i}(f)
$$




\section{Demonstraçāo :}

Para cada $i=1, \ldots, r$ seja $D_{i}$ um disco fechado centrado em $p_{i}$ contido em $D$ de modo que $D_{i} \cap D_{j}=\emptyset$ para $i \neq j$.

Seja $B=D-\cup_{i=1}^{r} \operatorname{int}\left(D_{i}\right)$ entào $\partial B=\partial D \cup\left(\cup_{i=1}^{r} \partial D_{1}\right)$.

Temos que $\frac{\partial f}{|\partial f|}$ é continua em $B$, portanto, pelo Lema 2.1, grau $\left.\frac{\partial f}{|\partial f|}\right|_{a B}=0$.

Como $\partial D_{i}$ tem orientação oposta a $\partial D$ tomemos $-\partial D_{i}$. Portanto,

$$
0=\left.\operatorname{grau}\left(\frac{\partial f}{|\partial f|}\right)\right|_{\lambda_{B}}=\left.\operatorname{grau}\left(\frac{\partial f}{|\partial f|}\right)\right|_{\partial D}-\left.\sum_{i=1}^{r} \operatorname{grau}\left(\frac{\partial f}{|\partial f|}\right)\right|_{\partial D,}
$$

e segue o resultado.

Teorema 2.4 Seja $D_{\varepsilon} \subset C^{n}$ tal que 0 é zero isolado de $\partial f$. Então para quase todo ponto a $\in \mathbf{C}^{n}$, suficientemente próximo de 0 , a equação $\partial f(z)-a=0$ possui exatamente $\mu_{g}(f)$ soluçōes dentro de $D_{\varepsilon}$.

\section{Demonstração:}

Pelo Teorema de Sard, o conjunto

$$
R=\left\{a \in \mathrm{C}^{r}: \text { a é valor regular de } \partial f\right\}
$$

é denso em $\mathrm{C}^{\pi}$.

Do fato de $a$ ser valor regular de $\partial f$, segue que $\partial f$ é localmente injetora numa vizinhança de $p \in \partial f^{-1}(a)$. Assim as soluçôes de $\partial f(\tilde{i})-a=0$ são pontos isolados e portanto formam um subconjunto finito de $D_{\varepsilon}$.

Seja $\left\{p_{1}, \ldots, p_{r}\right\}=\operatorname{int}\left(D_{\varepsilon}\right) \cap \partial f^{-3}(a)$. Tomemos $g(z)=f(z)-a z$. entào $p_{l}$ é ponto critico de $g$ e

$$
\left(\frac{\partial^{2} g\left(p_{l}\right)}{\partial z_{i} \partial z_{j}}\right)=\left(\frac{\partial^{2} f\left(p_{l}\right)}{\partial z_{i} \partial z_{j}}\right)
$$

é não singular para cada $l=1, \ldots, r$.

Portanto, pelo Teorema 2.3 cada solução tem multiplicidade 1 . Tomando-se os valores regulares $a$ de $\partial f$ que satisfazem $|a|<|\partial f(z)|$ para todo $z \in S_{\varepsilon}$ ( existem pois $|\partial f(z)|$ é limitado em $S_{\varepsilon}$ e $R$ é denso ). 
Pelo Lema 2.3 o número de soluçóes em $D_{\varepsilon}$ é igual ao $\left.\operatorname{grau}\left(\frac{\partial f(z)-a}{|\partial f(z)-a|}\right)\right|_{S_{\ell}=a D_{\ell}} \mathbf{e}$

$$
\left.\operatorname{grau}\left(\frac{\partial f(z)-a}{|\partial f(z)-a|}\right)\right|_{S_{s}}=\left.\operatorname{grau}\left(\frac{\partial f(z)}{|\partial f(z)|}\right)\right|_{S_{s}}=\mu_{g}(f)
$$

Portanto,

$$
\mu_{g}(f)=\operatorname{card}\left\{\partial f^{-1}(a) \cap D_{\varepsilon}: \text { a é valor regular de } \partial f \text { próximo de } 0\right\}
$$

Segue da demonstração que para uma pequena perturbação linear $f(z)+a z$ de $f(z)$ obtemos $\mu_{g}(f)$ pontos críticos não degenerados em uma vizinhança de 0 .

Definição 2.3 Uma aplicaşão $h: l \rightarrow \mathrm{C}$ é dita de Morse se h tem um número finito de pontos críticos $\left\{x_{1}, \ldots, x_{k}\right\}$ em $U, \epsilon x_{i}$ é ponto crítico nâo degenerado de $h, i=1, \ldots, k$. Quando as imagens dos pontos críticos sâo todas distintas, $h e^{\prime}$ estável $([7])$.

Teorema 2.5 Toda deformaçâo de Morse $f_{c}: U \rightarrow \mathbf{C}$ suficientemente próxima de $f$ tem exatamente $\mu_{g}(f)$ pontos críticos não degenerados.

\section{Demonstração :}

Seja $D \subset U^{\prime}$, um disco tal que 0 é o único ponto crítico de $\left.f\right|_{D}$.

Como $f$ e $f_{\varepsilon}$ sâo suficjentemente próximas, podemos supor que existe homotopia

$$
H_{t}(z)=t \frac{\partial f(z)}{|\partial f(z)|}+(1-t) \frac{\partial f_{\varepsilon}(z)}{\left|\partial f_{\varepsilon}(z)\right|}
$$

Pelo Teorema 2.1. temos que $\mu_{g}\left(f_{\varepsilon}\right)=\mu_{g}(f)$ ambas restritas à $\partial D$.

Sejam $\left\{x_{1}, \ldots, x_{\tau}\right\}$ pontos críticos nào degenerados de $f_{\varepsilon}$ no int $(D)$.

Basta portanto mostrar que $\mu_{g}(f)=r$.

Para cada $i=1, \ldots, r$ seja $D_{i}$ um disco fechado centrado em $x_{i}$ contido em $D$ de modo que $D_{i} \cap D_{j}=\emptyset$, para $i \neq j$. 
Seja $\mu_{g}^{i}\left(f_{\varepsilon}\right)$ o grau de $\left.\frac{f_{c}}{\left|f_{c}\right|}\right|_{\partial D_{\imath}}$. Como $x_{i}$ é não degenerado segue pelo Teorema 2.3 que $\mu_{g}^{i}\left(f_{\varepsilon}\right)=1$, mas pelo Lema 2.3 temos

$$
\mu_{g}(f)=\mu_{g}\left(f_{\varepsilon}\right)=\sum_{i=1}^{r} \mu_{g}^{i}\left(f_{\varepsilon}\right)=r
$$

Observação : A idéja central do teorema acima. de relacionar invariantes associados a uma singularidade com a geometria de uma perturbação estável, tem sido estudada recentemente por diversos autores [20] e [21]. 


\section{Capítulo 3}

\section{Caracterização Algébrica do Número de Milnor}

Neste capitulo será introduzido o conceito de multiplicidade algébrica, $\mu_{a}(f)$, de um germe holomorfo $f$ em 0 . Os resultados principais são os seguintes:

Teorema 3.1: $\mu_{a}(f)<\propto$ se, e somente se, 0 é singularidade isolada ou valor regular de $f$.

Teorema 3.2: $\mu_{a}(f)<\infty$ entâo existe difeomorfismo biholomorfo $h$ tal que $f \circ h$ é um polinômio.

Denotaremos por $\mathcal{O}_{n}$ o conjunto dos germes em 0 de funçôes holomorfas.

Temos que $\mathcal{O}_{n}$ é um anel local. cujo único ideal maximal, $\mathcal{M}_{n}$. é formado pelos germes de funçóes que se anulam em 0 .

Seja $J f$ o ideal de $\mathcal{O}_{n}$ gerado pelos germes em 0 das derivadas parciais $\frac{\partial f}{\partial z_{1}}$ para $i=1, \ldots, n$.

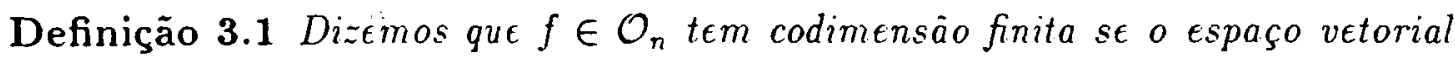

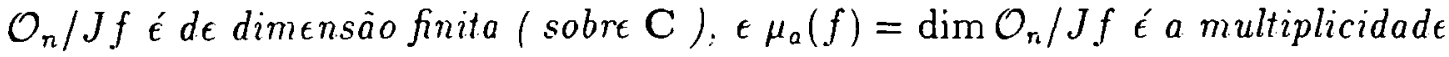
algébrica de $f \in \mathrm{\epsilon m} 0$.

Lema 3.1 Jf tem codimensâo finita se, e somente se, existe $k \geq 1$ tal que

$$
\mathcal{M}_{n}^{k} \subseteq J f
$$




\section{Demonstração :}

Suponhamos $J f$ de codimensão finita. Consideremos a seguinte sequência de ideais de $\mathcal{O}_{n}$.

$$
J f+\mathcal{M}^{o} \mathcal{O}_{n} \supseteq J f+\mathcal{M}_{n}^{1} \mathcal{O}_{n} \supseteq J f+\mathcal{M}_{n}^{2} \mathcal{O}_{n} \supseteq \ldots \supseteq J f
$$

Logo,

$$
\operatorname{dim} \frac{\mathcal{O}_{n}}{J f+\mathcal{M}_{n}^{k} \mathcal{O}_{n}} \leq \operatorname{dim} \frac{\mathcal{O}_{n}}{J f+\mathcal{M}_{n}^{k+1} \mathcal{O}_{n}}
$$

Do fato de $\operatorname{dim} \mathcal{O}_{n} / J f<\infty$, segue $\exists k \geq 1$ tal que

$$
J f+\mathcal{M}_{n}^{k} \mathcal{O}_{n}=J f+\mathcal{M}_{n}^{k+1} \mathcal{O}_{n}
$$

ainda,

$$
\mathcal{M}_{n}^{k} \mathcal{O}_{n} \subseteq J f+\mathcal{M}_{n}^{k} \mathcal{O}_{n}=J f+\mathcal{M}_{n}^{k+1} \mathcal{O}_{n}=J f+\mathcal{M}_{n}\left(\mathcal{M}_{n}^{k} \mathcal{O}_{n}\right)
$$

Portanto, pelo Lema de Nakayama [6], $\mathcal{M}_{n}^{k} \mathcal{O}_{n} \subseteq J f$.

Reciprocamente se $\exists k \geq 1, \mathcal{M}_{n}^{k} \mathcal{O}_{n} \subseteq J f$, entâo $\operatorname{dim} \mathcal{O}_{n} / J f \leq \operatorname{dim} \mathcal{O}_{n} / \mathcal{M}_{n}^{k}$.

Mostremos que $\mathcal{O}_{n} / \mathcal{M}_{n}^{k}$ é de dimensâo finita. Para tanto seja

$$
J^{k+1}: \mathcal{O}_{n} \rightarrow T_{o}^{k+1}
$$

onde $T_{0}^{k+1}$ é o conjunto dos polinômios de Taylor em 0 até ordem $k+1 \mathrm{e}$ $J^{k+1}(f)=j^{k+1} f=(k+1)$-jato de $f$.

Temos $J^{k+1}$ é um homomorfismo de anëis e $\operatorname{ker}\left(J^{k+1}\right)=\mathcal{M}_{n}^{k}$, logo

$$
\frac{\mathcal{O}_{n}}{\mathcal{M}_{n}^{k}} \simeq J^{k+1}\left(\mathcal{O}_{n}\right)=T_{0}^{k+1}
$$

Portanto, $\operatorname{dim} \mathcal{O}_{n} / \mathcal{M}_{n}^{k}$ é finito e segue o resultado.

Teorema 3.1 Seja $f: U \rightarrow \mathbf{C}, 0 \in U \subset \mathbf{C}^{n}$, então 0 é ponto crítico isolado ou ponto regular de $f$ se, e somente se, $\mu_{a}<\infty$. 


\section{Demonstração :}

Suponhamos que 0 é ponto crítico isolado.

Consideremos a subvariedade analitica

$$
V(J f)=\left\{z \in l: \frac{\partial f}{\partial z_{i}}(z)=0 ; i=1 \ldots, n\right\}
$$

Por hipótese. existe vizinhança (que podemos supor a própria $U$ ) tal que

$$
V(J f)=\{0\}
$$

Portanto, $I(V(J f))=\left\{h \in \mathcal{O}_{n}: h\right.$ se anula em $\left.V(J f)=0\right\}=\mathcal{M}_{n}$. Mas pelo Teorema de Zeros de Hilbert.

$$
\mathcal{M}_{n}=I(V(J f))=\operatorname{rad}(J f)=\left\{h \in \mathcal{O}_{n}: h^{k} \in J f \text { para algum } k \in \Lambda\right\}
$$

entào $J f \supseteq \mathcal{M}_{n}^{r}$ para algum $r \in \Lambda$.

Pelo Lema 3.1 segue que $\mu_{a}<\infty$.

Reciprocamente se $\mu_{a}<\infty$ entào pelo Lema $3.1 \exists k \geq 1$ tal que $\mathcal{M}_{n}^{k} \subseteq J f$. Logo, em particular, os monômios $z_{1}^{k}, \ldots, z_{n}^{k}$ são combinaçóes lineares de $\frac{\partial f}{\partial z_{1}}, \ldots, \frac{\partial f}{\partial z_{n}}$ com coeficientes em $\mathcal{O}_{n}$, ou seja,

$$
z_{i}^{k}=g_{i 1} \frac{\partial f}{\partial z_{1}}+g_{i 2} \frac{\partial f}{\partial z_{2}}+\ldots+g_{i n} \frac{\partial f}{\partial z_{n}} ; i=1 \ldots . . n
$$

Portanto, se $w=\left(u_{1}, \ldots, w_{n}\right)$ é ponto crítico de $f$, entào

$$
\frac{\partial f}{\partial \tilde{z}_{i}}(u)=0 \Rightarrow w_{1}^{k}=\ldots=u_{n}^{k}=0 \Rightarrow w_{1}=\ldots=w_{n}=0
$$

ou seja, $w=0$ é ponto crítico isolado de $f$.

Quando 0 é ponto regular. vemos facilmente que $\mathcal{O}_{n}=J f$ e, portanto, $\mu_{a}=0$.

Reciprocamente, se $\mu_{a}=0 J f$ tem elemento inversivel $\frac{\partial f}{\partial z_{1}}(0) \neq 0$ e portanto 0 é valor regular. 
Observação: No caso real. 0 pode ser ponto crítico isolado de $f$ mas $\operatorname{dim} \mathcal{E}_{n} / J f=\infty$, onde $\mathcal{E}_{n}$ é o conjunto dos germes em 0 de funçôes diferenciáveis de classe $C^{\infty}$.

Por exemplo, tomemos $f:\left(\mathbf{R}^{2}, 0\right) \rightarrow(\mathbf{R}, 0), f(x, y)=\left(x^{2}+y^{2}\right)^{2}$.

Entâo, 0 é singularidade isolada de $f$, mas não é singularidade isolada de sua complexificaşào $f_{c}:\left(\mathbf{C}^{2}, 0\right) \rightarrow($ C. 0$)$. Logo, $f_{c}$ é de codimensão infinita sobre $\mathbf{C}$, e portanto, também $f$ tem codimensâo infinita sobre $\mathbf{R}$.

O teorema a seguir nos diz que em se tratando de funçōes holomorfas com pontos críticos isolados nâo há perda de generalidade em considerar localmente a funçâo dada como um polinômio.

Teorema 3.2 Se $f: U \rightarrow$ C, $U$ vizinhança de $0 \mathrm{em} \mathrm{C}^{n}$, é holomorfa e $\mu_{a}<\infty$, entâo existe um inteiro $k$ tal que para todo germe $g$ com $j^{k} f=j^{k} g$, existe difeomorfismo biholomorfo $h$ tal que $g=f \circ h$.

\section{Demonstração :}

De $\mu_{a}<\propto \Rightarrow \exists k \geq 1$ tal que $\mathcal{M}_{n}^{k} \subseteq J f$.

Seja $g \in \mathcal{O}_{n}$ tal que $j^{k} g=j^{k} f$.

Observemos que $f-g \in \mathcal{M}_{n}^{k+1}$ e $f, g$ sào pontos no espaşo vetorial complexo $\mathcal{O}_{n}$. Assim, podemos definir

$$
F(z, t)=f_{t}(z)=(1-t) f(z)+t g(z) ; t \in[0,1]
$$

que é uma família de germes a um parâmetro, $f_{t}, \operatorname{com} f_{0}=f$ e $f_{1}=g$. Para cada $s$, iremos construir uma família $h_{t}$. de germes de difeomorfismos biholomorfos, com $t$ próximo de $s$, tal que

$$
f_{t} \circ h_{t}=f_{s}
$$

Isto é equivalente a mostrar a existencia de um germe em $(0, s)$ de uma aplicação holomorfa

$$
H: \mathbf{C}^{n} \times \mathbf{C} \rightarrow \mathbf{C}^{n},
$$

satisfazendo:

a) $H(z, s)=z$

b) $H(0, t)=0$ 
c) $F(H(z, t), t)=F(z, s)$

De fato, colocando $h_{t}(z)=H(z, t)$, a condiçào a) diz que $h_{s}=$ identidade. Logo, para $t$ próximo de $s, h_{t}$ é inversivel.

b) diz que $h_{t}$ leva a origem nela mesma.

Finalmente, $\mathbf{c}$ ) pode ser escrito como $f_{t} \circ h_{t}=f_{s}$

Notemos que por a) , c) é verdadeiro para $t=s$. Assim, a condiçào c) pode ser trocada pela afirmação que $F(H(\tilde{z}, t), t)$ nâo depende de $t$, isto é, a derivada com relaçào a $t$ é nula:

c') $\sum_{i=1}^{n} \frac{\partial F}{\partial z_{1}}(H(z, t), t) \frac{\partial H_{1}}{\partial t}(z, t)+\frac{\partial F}{\partial t}(H(z, t), t)=0$.

Logo, nosso problema é encontrar $H$ tal que a), b) e c') se verificam. Afirmamos que é suficiente construir um germe em (0.s) de uma aplicação holomorfa

$$
\xi: \mathbf{C}^{n} \times \mathbf{C} \rightarrow \mathbf{C}^{n} \text {, tal que }
$$

d) $\sum_{i=1}^{n} \xi_{i} \frac{\partial F}{\partial \tau_{i}}=-\frac{\partial F}{\partial t}$

e) $\xi_{i}(0 . t)=0$

Se existir tal $\xi$, este pode ser pensado como um campo vetorial em $\mathbf{C}^{n}$ dependendo do tempo. Assim. $\xi$ tem um fluxo. isto é, existe

$$
H: \mathbf{C}^{n} \times \mathbf{C} \rightarrow \mathbf{C}^{n} \text {. tal que }
$$

f) $\frac{\partial H}{\partial t}=\xi(H(z . t) \cdot t)$

e satisfazendo a condiçào inicial $H(z, s)=z$.

De fato, $\mathbf{c}^{\prime}$ ) pode ser trocado por $\mathbf{d}$ ) e f), pois

$$
\sum_{i=1}^{n} \frac{\partial F}{\partial z_{i}}(H, t) \frac{\partial H_{i}}{\partial t}+\frac{\partial F}{\partial t}(H, t) \stackrel{f}{=} \sum_{i}^{n} \frac{\partial F}{\partial z_{i}}(H, t) \xi_{i}(H . t)+\frac{\partial F}{\partial t}(H, t) \stackrel{\mathbf{d})}{=} 0
$$

b) pode ser trocado por a); e) ef)

Para $z=0$, temos a linha de fluxo (curva integral) $H(0, t)$ de $\xi$, isto é, $\frac{\partial H}{\partial t}(0, t)=\xi(H(0, t), t)$. Isto devido a $\left.\mathbf{f}\right)$ que garante a existência de fluxo local $\xi \mathrm{em} z=0$ satisfazendo a condição inicial $H(0, s)=0$. Notemos que e) diz 
que $\xi(0, t)=0=\frac{\partial 0}{\partial t}(0, t)$, com $0(0, s)=0$. Pela unicidade da linha de fluxo, $H(0, t)=0$.

Resta, portanto, estabelecer a existência de uma aplicação $\xi$ satisfazendo d) e e). $O$ argumento é algébrico e não depende da escolha de $s$. Logo, podemos supor $s=0$. Além disso, como trabalhamos simultaneamente com funçôes em $\mathcal{O}_{n}$ e $\mathcal{O}_{n+1}$, podemos tomar os elementos de $\mathcal{O}_{n}$ como sendo elementos de $\mathcal{O}_{n+1}$ independentes de $t$. Assim, a condição e) é o mesmo que $\xi_{i} \in \mathcal{M}_{n}$.

Ainda :

$$
\begin{gathered}
g-f \in \mathcal{M}_{n}^{k+1} \Rightarrow \frac{\partial}{\partial z_{i}}(g-f) \in \mathcal{M}_{n}^{k} \\
\frac{\partial F}{\partial t}=g-f \in \mathcal{M}_{n}^{k+1} \\
\frac{\partial F}{\partial z_{i}}=(1-t) \frac{\partial f}{\partial z_{i}}+t \frac{\partial g}{\partial z_{i}} \Rightarrow \frac{\partial F}{\partial z_{i}}-\frac{\partial f}{\partial z_{i}}=t \frac{\partial}{\partial z_{i}}(g-f)
\end{gathered}
$$

Como $\left(z_{1}, \ldots, z_{n}, t\right) \mapsto t \in \mathcal{M}_{n+1}$. segue que

$$
\frac{\partial F}{\partial z_{i}}-\frac{\partial f}{\partial z_{i}} \in \mathcal{M}_{n+1} \mathcal{M}_{n}^{k}
$$

Portanto, $<\frac{\partial f}{\partial z_{1}}>\subseteq<\frac{\partial F}{\partial z_{1}}>+\mathcal{M}_{n+1} \mathcal{M}_{n}^{k}$ donde,

$$
\mathcal{M}_{n}<\frac{\partial f}{\partial \tilde{z}_{i}}>\subseteq \mathcal{M}_{n}<\frac{\partial F}{\partial \tilde{z}_{i}}>+\mathcal{M}_{n+1} \cdot \mathcal{M}_{n}^{k+1}
$$

Então,

$\mathcal{M}_{n}^{k+1}=\mathcal{M}_{n} \mathcal{M}_{n}^{k} \subseteq \mathcal{M}_{n} J f=\mathcal{M}_{n}<\frac{\partial f}{\partial z_{i}}>\subseteq \mathcal{M}_{n}<\frac{\partial F}{\partial z_{i}}>+\mathcal{M}_{n+1} \mathcal{M}_{n}^{k+1}$

Pelo Lema de Nakayama obtemos;

$$
\mathcal{M}_{n}^{k+1} \subseteq \mathcal{M}_{n}<\frac{\partial F}{\partial z_{i}}>
$$


Do fato de $-\frac{\partial F}{\partial t} \in \mathcal{M}_{n}^{k+1}$, segue que

$$
-\frac{\partial F}{\partial t}=\sum_{i=1}^{n} \xi_{i} \frac{\partial F}{\partial z_{i}}
$$

o que mostra a validade de d) completando a demonstração.

Observemos que se $g$ é o polinômio de Taylor de $f$ em 0 até ordem $k$ então $f \circ h=g$ é um polinômio.

Definição 3.2 Um germe $f$ que satisfaz $\mu_{a}<\infty$ é dito finitamente determinado.

Exemplo 3.1 Calcular $\mu_{a}$ de $f:\left(\mathbf{C}^{2}, 0\right) \rightarrow(\mathbf{C}, 0)$ dada por:

$$
f(z, w)=z^{2} w+w^{4}
$$

Temos que $J f=<2 z w, \tilde{z}^{2}+4 w^{3}>$. Logo $z^{i} w^{j} \in J f$ para $i \neq 0$ e $j \neq 0$. $z^{3} \in J f$, pois $z^{3}+4 w^{3} z \in J f$ e $4 w^{3} z \in J f$ $w^{4} \in J f$, pois $z^{2} w+4 w^{4} \in J f$ e $z^{2} w \in J f$

Assim. $z^{j} \in J f$ para $j \geq 3$ e $u^{j} \in J f$ para $j \geq 4$.

Observamos que $1, z, w, z^{2}, u^{2}, w^{3} \notin J f$. Portanto.

$$
\frac{\mathcal{O}_{2}}{J f}=<1, z, w, z^{2}, w^{2}>
$$

ou seja, $\mu_{o}=5$. 


\section{Capítulo 4}

\section{Equivalência das Definições}

O objetivo deste capítulo é demonstrar a equivalência entre os conceitos definidos anteriormente. Isto será feito em duas etapas: No Teorema 4.1, será obtida a equivalência entre a multiplicidade geométrica e o número de Milnor, no Teoreza 4.2 , mostraremos que a multiplicidade geométrica e a multiplicidade algébrica coincidem.

\subsection{Equivalência entre a Multiplicidade Geo- métrica e o Número de Milnor}

Teorema $4.1 \mu_{g}=$ posto $H_{n-1}\left(F_{\theta}, \mathbf{Z}\right)$.

Antes de demonstrarmos este teorema precisamos de una fórmula que relacione o grau de uma aplicação $v: S^{k} \rightarrow S^{k}$ com a topologia de uma subvariedade de $S^{k}$.

Seja .: uma variedade fechada e $h: M \rightarrow M$ uma aplicaçâa. Entâo para cada $k$ existe um homomorfismo induzido a nivel de homologia com coeficientes racionais

$$
h_{k}: H_{k}(M ; \mathbf{Q}) \rightarrow H_{k}(M ; \mathbf{Q})
$$

Para cada $k$, nós podemos escolher uma base finita para o espaço vetorial racional $B_{k}(M ; \mathbf{Q})$ e escrever $h_{k}$ como uma matriz com relação a esta base. Denotamos For $\operatorname{Tr}\left(h_{k}\right)$ o traço desta matriz. 
Definição 4.1 número de Lefschetz de $h$ é $d \epsilon$ finido por

$$
L(h)=\sum_{k=0}(-1)^{k} \operatorname{Tr}\left(h_{k}\right)
$$

Observemos que $L(h)$ é independente da escolha envolvida e, portanto, está bem definido. Claro que $L(h)$ depende somente da classe de homotopia de $h$; pois se $g$ e $h$ são homotópicos entào $g_{*}=h_{*}$, isto é, as induzidas sào iguais. No caso em que $M$ é uma variedade $C^{\infty}$ orientada entào, pode-se mostrar [12, pag. 420] que

$$
L(h)=I(\Delta(M), \operatorname{graf}(h))
$$

onde

$$
\begin{gathered}
\operatorname{graf}(h)=\{(x, y) \in M \times M: y=h(x)\} \mathrm{e} \\
\Delta(M)=\{(x, y) \in M \times M: y=x\}
\end{gathered}
$$

Observemos que

$$
\operatorname{graf}(h) \cap \Delta(M)=F i x(h)=\{x \in M: h(x)=x\}
$$

e que podemos supor (fazendo uma homotopia) que graf $f(h)$ é transversal à $\Delta(M)$.

Portanto temos

$$
\sum_{x \in F i x(h)} \operatorname{sinal}\left(d h_{x}-I d\right)=I(\Delta(M), g r a f(h))=L(h)
$$

onde Id é a aplicaçào identidade.

Definição 4.2 A característica de Euler $d \epsilon M: \chi(M)$. $\epsilon$ definida por

$$
\chi(M)=\sum_{j}(-1)^{j} \text { posto }_{j}(M) .
$$

Por outro lado temos:

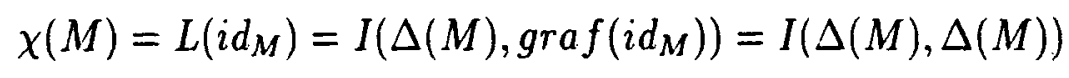


Lema 4.1 Sejam v: $S^{k} \rightarrow S^{k}$ uma aplicação $C^{\infty} \in M \subset S^{k}$ uma regiâo de $S^{k}$ com bordo suave. Suponhamos que:

1) Fix(v) $\subset M$

2) $v(x) \neq-x, \forall x \in M$

3) $\left\langle v(x), n(x)>>0\right.$ para todo $x \in \partial M$ onde $n(x) \in T_{x} S^{k} \dot{\epsilon}$ um vetor normal $\dot{a}$ $\partial M$ que aponta para o interior de $M(<,>$ produto interno euclidiano).

Então

$$
\chi(M)=1+(-1)^{k} \operatorname{grau}(v)
$$

\section{Demonstração:}

Perturbando $v$, se necessário, podemos supor que $v$ tem pontos fixos isolados.

Para v: $S^{k} \rightarrow S^{k}$ temos:

$$
L(v)=\sum_{j}(-1)^{j} \operatorname{Tr}\left(v_{j}: H_{j}\left(S^{k}\right) \rightarrow H_{j}\left(S^{k}\right)\right)=1+(-1)^{k} \operatorname{grau}(v)
$$

De fato,

$$
\sum_{j}(-1)^{j} \operatorname{Tr}\left(v_{j}: H_{j}\left(S^{k}\right) \rightarrow H_{j}\left(S^{k}\right)\right)=1+(-1)^{k} \operatorname{Tr}\left(v_{k}: H_{k}\left(S^{k}\right) \rightarrow H_{k}\left(S^{k}\right)\right) .
$$

Agora, seja $[z]$ o gerador de $H_{k}\left(S^{k}\right)$. Então $v_{k}[z]=\operatorname{Tr}\left(v_{k}\right)[z]=\operatorname{grau}(v)[z]$.

Da hipótese 2), podemos definir $v_{t}: M \rightarrow S^{k}$ por

$$
v_{t}(x)=\frac{(1-t) x+t v(x)}{|(1-t) x+t v(x)|}
$$

De fato, se $\exists y$ tal que $(1-t) y+t v(y)=0 \Rightarrow(1-t) y=-t v(y)$ $\Rightarrow|1-t|=|t| \Rightarrow t=1 / 2 \Rightarrow v(y)=-y$, o que é uma contradição.

Afirmamos que para $t$ suficientemente pequeno $v_{t}(M) \subset M$.

De fato, podemos construir uma cobertura de $M$ por abertos $B_{\varepsilon}(x)$ e $U_{x}$, $x \in M$ como segue:

1) se $x \in \operatorname{int}(M)$.

Dado $\varepsilon>0$, seja $B_{\varepsilon}(x)=\{y:|x-y|<\varepsilon\} \cap M$ 
Pela continuidade de $v_{t}(x)$ com relaşâo a $t, \exists \delta_{x}>0$ tal que $0 \leq t \leq \delta_{x} \Rightarrow$ $v_{t}(x) \subset B_{\varepsilon}(x)$.

2) se $x \in \partial M$.

Consideremos a curva $\alpha(t)=v_{t}(x)$ com $\alpha(0)=x$. Pela hipótese 3 ) segue que

$$
<\alpha^{\prime}(0), n(x)>>0
$$

Da continuidade do produto interno, $\exists \lambda_{x}>0$ tal que $\left\langle\alpha^{\prime}(t), n(x)>>0\right.$ para $0 \leq t \leq \lambda_{x}$, ou seja, $a(t)=v_{t}(x) \subset M$.

Para cada $x \in \partial M$, seja $U_{x}$ vizinhança aberta de $M$, tal que

$$
\alpha(t) \subset U_{x} \subset M, 0 \leq t \leq \lambda_{x}
$$

Como $M$ é compacta, podemos extrair uma subcobertura finita, isto é,

$$
M=U_{x_{1}} \cup \ldots \cup U_{x_{r}} \cup B_{\varepsilon}\left(y_{1}\right) \cup \ldots \cup B_{\varepsilon}\left(y_{k}\right)
$$

Portanto, para $\delta=\min \left\{\delta_{x_{1}}, \ldots, \delta_{x_{r}}, \lambda_{y_{1}}, \ldots, \lambda_{y_{k}}\right\}$ e $0 \leq t \leq \delta$, temos

$$
v_{t}(M) \subset M
$$

Logo, pela invariância por homotopia do número de Lefschttz temos para $0 \leq t \leq \delta$

$$
L\left(v_{t}\right)=L\left(v_{o}\right)=L\left(i d_{M}\right)=\chi(M)
$$

Afirmamos que $F i x\left(v_{t}\right)=F i x(v)$ para todo $t>0$.

De fato, claro que $F i x(v) \subseteq F i x\left(v_{t}\right)$. Por outro lado, se $y \in F i x\left(v_{t}\right)$, entào

$$
\frac{(1-t) y+t v(y)}{|(1-t) y+t v(y)|}=y
$$

$\Rightarrow t v(y)=y[|(1-t) y+t v(y)|-(1-t)] \Rightarrow||(1-t) y+t v(y)|-(1-t)|=|t| \Rightarrow$ $c=|(1-t) y+t v(y)|= \pm t+1-t \Rightarrow c=1$ ou $c=1-2 t$.

Se $c=1 \Rightarrow t v(y)=t y \Rightarrow y \in F i x(v)$

Se $c=1-2 t \Rightarrow t v(y)=-t y \Rightarrow v(y)=-y$, o que é uma contradição pela hipótese 2). 
Assim, Fix $\left(v_{t}\right) \subseteq$ Fix $(v)$. Portanto,

$$
F i x\left(v_{t}\right)=F i x(v)
$$

Além disso, o sinal $\left(d v_{t}(x)-I d\right)$ para $x \in F i x\left(v_{t}\right)$ é um inteiro que varia continuamente com $t$, isto implica que $\operatorname{sinal}\left(d v_{t}(x)-I d\right)=\operatorname{sinal}(d v(x)-I d)$. Portanto,

$L(v)=\sum_{x \in F i x(v)} \operatorname{sinal}(d v(x)-I d)=\sum_{x \in F i x\left(v_{t}\right)} \operatorname{sinal}\left(d v_{t}(x)-I d\right)=L\left(v_{t}\right)=$ $\chi(M)$. Logo,

$$
\chi(M)=1+(-1)^{k} \operatorname{grau}(v)
$$

Lema 4.2 As hipóteses do Lema 4.1 se verificam para v: $S_{\varepsilon} \rightarrow S_{\varepsilon}$ definida por

$$
\begin{gathered}
v(z)=\varepsilon \frac{\operatorname{grad} f(z)}{|\operatorname{grad} f(z)|} \text { e } \\
M=\left\{z \in S_{\varepsilon}: \operatorname{Re}(f(z)) \geq 0\right\},
\end{gathered}
$$

onde $\operatorname{Re}(f(z))$ é a parte real de $f(z)$.

A demonstração do lema 4.2 depende ainda do seguinte resultado:

Lema 4.3 O conjunto dos pontos críticos $d \epsilon .\left.f\right|_{\epsilon}$ é dado por

$$
\left\{z \in S_{\varepsilon}: \operatorname{grad} f(z)=c z, c \in \mathbf{C}\right\}
$$

$\epsilon$ para $\varepsilon$ suficientemente pequeno $K$ nâo contém pontos críticos $d \epsilon f \mid S_{\varepsilon}$.

A demonstração desse lema pode ser encontrada em [1, pag. 55].

\section{Demonstração do lema 4.2:}

Podemos escrever $M=\cup_{-\pi / 2 \leq \theta \leq \pi / 2} F_{\theta} \cup K_{\varepsilon}$ de maneira que $\partial M=F_{-\pi / 2} \cup K_{\varepsilon} \cup F_{\pi / 2}$ é $C^{\infty}$, pelo Lema 1.4.

Hipótese 1): $z \in F i x(v) \Leftrightarrow v(z)=z \Leftrightarrow \operatorname{grad} f(z)=\lambda z \operatorname{com} \lambda>0$, então pelo Lema 4.3 segue que $f(z) \neq 0$. Logo, $\operatorname{grad} \log (f(z))=\frac{\lambda z}{f(z)}$ e pelo Lema 1.2 segue que $\operatorname{Re}\left(\frac{\lambda}{f(z)}\right)>0$. Como $\lambda>0$ temos $\operatorname{Re}(f(z))>0$, ou seja $z \in \operatorname{int}(M)$. 
Hipótcse 2): É verificada analogamente. Se $v(z)=-z$ entâo grad $f(z)=c z$ com $c<0$. Isto significa que grad $\log (f(z))=\frac{c z}{f(z)} \operatorname{com} \operatorname{Re}\left(\frac{f}{f(z)}\right)>0$, ou seja $R e(f(z))<0$ e $z \notin M$.

Hipótese 3): Seja $p(t)$ uma curva em $S_{\varepsilon}$ passando pelo ponto $z \in \partial M \mathrm{e}$ transversal a $\partial M$ tal que $p(0)=z, p^{\prime}(0)=n(z)$ e $R \epsilon(f(p(t))$ seja crescente. Então $\frac{d}{d t} \operatorname{Re}\left(\left.f(p(t))\right|_{t=0}>0\right.$.

Derivando obtemos

$$
\left.\frac{d}{d t} f \circ p(t)\right|_{t=0}=<p^{\prime}(t), \text { grad } f(p(t))>\left.\right|_{t=0}=<n(z), \operatorname{grad} f(z)>
$$

portanto, $\operatorname{Re}(<n(z), \operatorname{grad} f(z)>)>0$.

\section{Demonstração do Teorema 4.1:}

Por definição $\mu_{g}=\operatorname{grau}\left(\frac{\partial f}{|\partial f|}\right)$ onde $\frac{\partial f}{|\partial f|}: S_{\varepsilon} \rightarrow S_{1}$ e

$$
\frac{\partial f}{|\partial f|}(z)=\frac{\partial f}{|\overline{\partial f}|}(z)=\frac{\partial f(z)}{|\operatorname{grad} f(z)|}
$$

Se $\varphi\left(z_{1}, \ldots, z_{n}\right)=\left(\overline{z_{1}}, \ldots, \overline{z_{n}}\right)$ entào

$$
\frac{\partial f}{|\partial f|}=\varphi \circ \frac{\operatorname{grad} f}{|\operatorname{grad} f|}
$$

Como o grau da composta é igual ao produto dos graus, segue que

$$
\mu_{s}=(\operatorname{grau} \varphi)\left(\operatorname{grau} \frac{\operatorname{grad} f}{|\operatorname{grad} f|}\right)=(-1)^{n} \operatorname{grau}\left(\frac{\operatorname{grad} f}{|\operatorname{grad} f|}\right)
$$

Sejam $v: S_{\varepsilon} \rightarrow S_{\varepsilon}$ e $M=\left\{z \in S_{\varepsilon}: R \epsilon(f(z)) \geq 0\right\}$ onde

$$
v(z)=\varepsilon \frac{\operatorname{grad} f(z)}{|\operatorname{grad} f(z)|}
$$

Pelo lema 4.2, temos que $M$ satisfaz as hipóteses do Lema 4.1 portanto,

$$
\chi(M)=1+(-1)^{2 n-1} \operatorname{grau}(v)
$$


Além disso, $\phi_{\varepsilon}: \operatorname{int}(M) \rightarrow C$ é uma fibraçào cuja fibra típica é $F_{\theta}$ onde $C$ é o semi-círculo dado por $C=\left\{e^{i \theta}:-\pi / 2<\theta<\pi / 2\right\}$, portanto é possível exprimir a característica de Euler de $M$ em termos das características de Euler da fibra e da base [11, pag. 322]

$$
\chi(M)=\chi\left(F_{\theta}\right) \lambda(C)=\chi\left(F_{\theta}\right)=\sum_{j}(-1)^{j} \operatorname{postoH}_{j}\left(F_{\theta}\right)
$$

Pelo Teorema 1.5 temos:

$$
\lambda(M)=1+(-1)^{n-1} \text { posto }_{n-1}\left(F_{\theta}\right)
$$

Por (4.1) e (4.2) temos:

$$
1+(-1)^{2 n-1} \operatorname{grau}(v)=1+(-1)^{n-1} \text { posto }_{n-1}\left(F_{\theta}\right)
$$

Como grau $(v)=(-1)^{-n} \mu_{g}$, segue que

$$
(-1)^{2 n-1}(-1)^{-n} \mu_{s}=(-1)^{n-1} \text { posto }_{n-1}\left(F_{\theta}\right)
$$

Logo, $\mu_{9}=$ posto $_{n-1}\left(F_{\theta}\right)$.

\subsection{Equivalência entre a Multiplicidade Algé- brica e a Multiplicidade Gradiente}

Teorema $4.2 \mu_{a}=\mu_{g}$.

Denotamos por $\mathcal{O}_{n, z}=\mathcal{O}_{z}$ o anel dos germes em 0 das funçôes holomorfas, onde $z=\left(z_{1}, \ldots, z_{n}\right)$, indicam as coordenadas em $\mathbf{C}^{n}$, e $\mathcal{O}_{y}$ o mesmo anel com coordenadas $\left(y_{1}, \ldots, y_{n}\right)$ no contradomínio. Seja,

$$
\partial f=\left(\frac{\partial f}{\partial z_{1}}, \ldots, \frac{\partial f}{\partial z_{n}}\right):\left(\mathbf{C}^{n}, 0\right) \rightarrow\left(\mathbf{C}^{n}, 0\right)
$$


observemos que o homomorfismo $(\partial f)^{*}: \mathcal{O}_{y} \rightarrow \mathcal{O}_{z}$ definido por:

$$
(\partial f)^{*}(\varphi)=\varphi \circ \partial f
$$

torna $\mathcal{O}_{z}$ um $\mathcal{O}_{y}$-módulo.

Lema $4.4 \mathcal{O}_{z}$ é um $\mathcal{O}_{y}$-módulo gerado por $\mu_{a}$ elementos.

Observação: Poderíamos obter este resultado diretamente do Teorema de Preparação de Malgrange [7]. No entanto, a demonstraçâo apresentada usa o Teorema de Preparaçâo de W'eierstrass [7] e é mais adequada para relacionar $\mu_{a}$ com o número de soluçào de $\partial f(z)-a$, para $a$ valor regular suficientemente próximo de 0 .

\section{Demonstração do Lema 4.4:}

Consideremos

$$
V=\left\{\left(y_{1}, \ldots, y_{n}, z_{1}, \ldots, z_{n}\right) \in \mathbf{C}^{n} \times \mathbf{C}^{n}: y_{j}=\frac{\partial f}{\partial z_{j}}\left(z_{1}, \ldots, z_{n}\right)\right\}=\operatorname{graf}(\partial f)
$$

Seja $\mathcal{A}=I d(V) \circ$ ideal de $\mathcal{O}_{y, z}$ gerado por $\left\{y_{j}-\frac{\partial \mathcal{f}}{\partial z}\left(z_{1}, \ldots, z_{n}\right)\right\}, j=1, \ldots, n$.

Afirmamos que $\mathcal{A}$ é um jeal primo.

De fato, seja

$$
H: \mathbf{C}^{n} \times \mathbf{C}^{n} \rightarrow \mathbf{C}^{n} \times \mathbf{C}^{n}
$$

onde $H(y, z)=(Y, z)$ e $Y_{j}=y_{j}-\frac{\partial f}{\partial z}$. Temos que $H$ é um difeomorfismo e, portanto

$$
H^{*}: \mathcal{O}_{Y, z} \rightarrow \mathcal{O}_{y, z}
$$

definido por $H^{*}(h)=h \circ H$. é um isomorfismo de anéis.

Seja $\phi: \mathbf{C}^{n} \rightarrow \mathbf{C}^{n} \times \mathbf{C}^{n}$ dada por $\phi(z)=(\partial f(z), z) \mathrm{e}$

$$
\phi^{*}: \mathcal{O}_{y, z} \rightarrow \mathcal{O}_{z}
$$

sua induzida. Entâo, $(H \circ \phi)^{*}(g)=g(H \circ \phi)=g(0, z)$

Observemos que:

1) $\operatorname{Ker}(H \circ \phi)^{*}=<Y_{1}, \ldots, Y_{n}>=\left(H^{*}\right)^{-1}(\mathcal{A})$. 
De fato, $\supseteq$ é imediata. Para $\subseteq$, basta usar o Lema de Hadamard.

2) $(H \circ \phi)^{*}=\phi^{*} \circ H^{*}$

3) $(H \circ \phi)^{*}$ é um isomorfismo de $\mathcal{O}_{Y_{,}} /\left(\operatorname{Ker}(H \circ \phi)^{*}=\left(H^{*}\right)^{-1}(\mathcal{A})\right)$ e $\mathcal{O}_{z}$.

Como $(H \circ \phi)^{*} \circ\left(H^{*}\right)^{-1}=\phi^{*}$, segue que $\phi^{*}\left(\mathcal{O}_{y, z} / \mathcal{A}\right)$ é um isomorfismo, pois

$$
\mathcal{O}_{y, z} \stackrel{\left(H^{*}\right)^{-1}}{\simeq} \mathcal{O}_{Y, z} \Rightarrow \frac{\mathcal{O}_{y, z}}{\mathcal{A}} \simeq \frac{\mathcal{O}_{Y, z}}{\left(H^{*}\right)^{-1}(\mathcal{A})} \simeq \mathcal{O}_{z}
$$

Portanto $\frac{\mathcal{O}_{y, z}}{\mathcal{A}} \simeq \mathcal{O}_{z}$.

Como $\mathcal{O}_{z}$ é anel de integridade, segue que $\mathcal{A}$ é um ideal primo.

Sendo 0 singularidade isolada de $f$, existe $j$ tal que $\frac{\partial f}{\partial z_{j}}\left(0, \ldots, 0, z_{n}\right) \neq 0$. Como

$$
\frac{\partial f}{\partial z_{j}}(0)=0, \text { tem-se que } \frac{\partial f}{\partial z_{j}}\left(0, \ldots, 0, z_{n}\right)=z_{n}^{k} g\left(z_{n}\right), \operatorname{com} g(0) \neq 0
$$

Portanto, pelo Teorema de Preparação de Weierstrass, existe uma função holomorfa $h$ definida numa vizinhança de $0 \mathrm{em} \mathbf{C}^{n} \times \mathbf{C}$ tal que

$$
h \frac{\partial f}{\partial z_{j}}=g_{n}=z_{n}^{k}+a_{1} z_{n}^{k-1}+\ldots+a_{k}
$$

onde $a_{j}$ são funções holomorfas em $\left(y_{1}, \ldots, y_{n}, z_{1}, \ldots, z_{n-1}\right)$. Como $\mathcal{A}$ é um ideal, $g_{n} \in \mathcal{A}$. Logo, $\tilde{z_{n}}=$ classe de $z_{n}$ em $\mathcal{O}_{y, z} / \mathcal{A}$ satisfaz uma equação algébrica com coeficientes em $\mathcal{O}_{y, z_{1}, \ldots, z_{n-1}} /\left(\mathcal{A} \cap \mathcal{O}_{y, z_{1}, \ldots, z_{n-1}}\right)$.

Além disso, pelo Teorema de Divisão de Weierstrass, se $g \in \mathcal{O}_{y, z}$ então $g=q g_{n}+r$ onde $r \in \mathcal{O}_{y_{1, z_{1}}, \ldots, z_{n-1}}\left[z_{n}\right]$

Logo, $g \equiv r \bmod \mathcal{A}$, ou seja,

$$
\frac{\mathcal{O}_{y, z}}{\mathcal{A}} \simeq \frac{\mathcal{O}_{y, z_{1}, \ldots, z_{n-1}}}{\mathcal{A} \cap \mathcal{O}_{y, z_{1}, \ldots, z_{n-1}}}[\tilde{z}]
$$

Procedendo analogamente com os ideais $\mathcal{A} \cap \mathcal{O}_{y, z_{1}, \ldots, z_{n-1}}$ sucessivamente $\mathrm{e}$ usando o fato que $f$ é de codimensão finita obtemos

$$
\frac{\mathcal{O}_{y, z}}{\mathcal{A}} \simeq \frac{\mathcal{O}_{y, z_{1}, \ldots, z_{n-1}}}{\mathcal{A} \cap \mathcal{O}_{y, z_{1}, \ldots, z_{n-1}}}\left[\tilde{z_{n}}\right] \simeq \ldots \simeq \frac{\mathcal{O}_{y}}{\mathcal{A} \cap \mathcal{O}_{y}}\left[z_{1}, \ldots, \tilde{z_{n}}\right] \simeq \mathcal{O}_{y}\left[\tilde{z_{1}}, \ldots, \tilde{z}_{n}\right]
$$

Portanto, usando o isomorfismo $(\phi)^{*}\left(\mathcal{O}_{y, z} / \mathcal{A}\right) \simeq \mathcal{O}_{z}$, obtemos $\mathcal{O}_{z} \simeq \phi^{*}\left(\mathcal{O}_{y, z} / \mathcal{A}\right) \simeq \phi^{*}\left(\mathcal{O}_{y}\left[\tilde{z_{1}}, \ldots, \tilde{z_{n}}\right]\right)=\phi^{*}\left(\mathcal{O}_{y}\right)\left[\tilde{z_{1}}, \ldots, \tilde{z_{n}}\right]=(\partial f)^{*}\left(\mathcal{O}_{y}\right)\left[\tilde{z_{1}}, \ldots, \tilde{z_{n}}\right]$. 
Isto é, $\mathcal{O}_{z}$ é uma extensão algébrica finita do subanel $(\partial f)^{*}\left(\mathcal{O}_{y}\right)$. Portanto $\mathcal{O}_{z}$ é um $(\partial f)^{*}\left(\mathcal{O}_{y}\right)$-módulo finitamente gerado.

Temos que $\operatorname{dim} \mathcal{O}_{z} / J f=\mu_{a}$. Sejam $\left\{b_{1}, \ldots, b_{\mu_{a}}\right\}$ uma base de $\mathcal{O}_{z} / J f$ e $\left\{c_{1}, \ldots, c_{\mu_{a}}\right\}$ elementos de $\mathcal{O}_{z}$ tal que $\pi\left(c_{j}\right)=b_{j}$, onde

$$
\pi: \mathcal{O}_{z} \rightarrow \mathcal{O}_{z} / J f
$$

é a projeçào canônica.

Seja $\beta \circ(\partial f)^{*}\left(\mathcal{O}_{y}\right)$-módulo gerado por $\left\{c_{1}, \ldots, c_{\mu_{a}}\right\}$.

Entâo $\mathcal{O}_{z}=\beta+J f \mathcal{O}_{z}$, pois se $p \in \mathcal{O}_{z} \Rightarrow \pi(p)=r_{1} b_{1}+\ldots+r_{\mu_{a}} b_{\mu_{a}}=$ $r_{1} \pi\left(c_{1}\right)+\ldots+r_{\mu_{a}} \pi\left(c_{\mu_{o}}\right) \Rightarrow p=r_{1} c_{1}+\ldots+r_{\mu_{a}} c_{\mu_{o}}+s$ onde $s \in J f$.

Portanto $\mathcal{O}_{z}=\beta+J f \mathcal{O}_{z}$ e, pelo Lema de Nakayama segue que $\mathcal{O}_{z}=\beta$, ou seja $\mathcal{O}_{z}$ é um $(\partial f)^{*}\left(\mathcal{O}_{y}\right)$-módulo gerado por $\mu_{a}$ elementos.

Passemos à demonstração do Teorema 4.2.

Usando o fato de que $\left\{\frac{\partial f}{\partial z_{1}}, \ldots, \frac{\partial f}{\partial z_{n}}\right\}$ é um sistema de parâmetros é possivel obter-se que $\mathcal{O}_{z}$ é um $\mathcal{O}_{y}$-módulo livre de posto $\mu_{a}$ [14].

$\mathrm{Na}$ sequência, indicaremos por $K\{D\}$ corpo de fraçôes de um anel de integridade $D$.

Como $\mathcal{A}$ é um ideal primo podemos formar o corpo quociente $\hat{K}=K\left\{\mathcal{O}_{y, z} / \mathcal{A}\right\}$ e do fato de cada $\tilde{z}_{j}$ ser algébrico sobre $\mathcal{O}_{y}$ obtemos que

$$
K\left\{\mathcal{O}_{y}\left[\tilde{z_{1}}, \ldots, \tilde{z_{n}}\right]\right\}=K\left\{\mathcal{O}_{y}\right\}\left[\tilde{z_{1}}, \ldots, \tilde{z_{n}}\right]
$$

Assim, podemos escrever

$$
\begin{gathered}
K_{z}=K^{\prime}\left\{\mathcal{O}_{z}\right\} \simeq K\left\{\mathcal{O}_{y, z} / \mathcal{A}\right\} \simeq K\left\{(\partial f)^{*}\left(\mathcal{O}_{y}\right)\left[\tilde{z_{1}}, \ldots, \hat{z_{n}}\right]\right\}= \\
=K\left\{(\partial f)^{*}\left(\mathcal{O}_{y}\right)\right\}\left[\tilde{z_{1}}, \ldots, \tilde{z_{n}}\right]=(\partial f)^{*} K\left\{\mathcal{O}_{y}\right\}\left[\tilde{z_{1}}, \ldots, \tilde{z_{n}}\right]=(\partial f)^{*} K_{y}\left[\tilde{z_{1}}, \ldots, \tilde{z_{n}}\right] .
\end{gathered}
$$

Portanto, $K_{z} \simeq(\partial f)^{*} K_{y}\left[\tilde{z_{1}}, \ldots, \tilde{z_{n}}\right]$ e pelo Teorema do Elemento Primitivo [13] existe uma combinação linear $\xi=c_{1} \tilde{z_{1}}+\ldots+c_{n} \tilde{z_{n}}$ tal que

$$
K_{z} \simeq(\partial f)^{*}\left(K_{y}\right)[\xi]
$$


Fazendo uma mudança de coordenadas podemos supor $\xi=z_{1}$ de modo que

$$
K_{z} \simeq(\partial f)^{*}\left(K_{y}\right)\left[z_{1}\right]
$$

Seja $p$ polinômio irredutível com coeficientes em $(\partial f)^{*}\left(K_{y}\right)$ tal que $p\left(z_{1}\right)=0$. Como $z_{1}$ é combinaçâo linear dos $\hat{z}_{j}, j=1, \ldots, n$, temos que $z_{1}$ satisfaz uma equação polinomial com coeficientes em $(\partial f)^{*}\left(\mathcal{O}_{y}\right)$. Logo cada coeficiente de $p$ também satisfaz uma tal equaçào [13, pag. 240].

Mas como $(\partial f)^{*}\left(\mathcal{O}_{y}\right)$ é integralmente fechado, por ser dominio de fatorizaçào única, isto implica que cada coeficiente de $p$ pertence a $(\partial f)^{*}\left(\mathcal{O}_{y}\right)$.

Temos que $\left[K_{z}=(\partial f)^{*}\left(K_{y}\right)\left[z_{1}\right]:(\partial f)^{*}\left(K_{y}\right)\right]=\mu_{a}=$ grau de $p$.

De fato, suponhamos que grau de $p=d$. Sabemos que $\left\{1, z_{1}, \ldots, z_{1}^{d-1}\right\}$ é uma base de $K_{z}$ sobre $K_{y}^{\prime}$, logo são linearmente independentes sobre $(\partial f)^{*}\left(K_{y}\right)$ e, portanto linearmente independentes sobre $(\partial f)^{*}\left(\mathcal{O}_{y}\right)$.

Como $\mathcal{O}_{z}$ é um $\mathcal{O}_{y}$-módulo livre de posto $\mu_{a}$, segue que $d \leq \mu_{a}$.

Seja $\left\{\alpha_{1}, \ldots, \alpha_{\mu_{a}}\right\}$ base de $\mathcal{O}_{z}$ como $\mathcal{O}_{y}$-módulo livre. Sejam $a_{i}, b_{i} \in \mathcal{O}_{y}$ temos que

$$
\sum_{i=1}^{\mu_{a}} \frac{a_{i}}{b_{i}} \alpha_{i}=0 \Leftrightarrow a_{i}=0
$$

ou seja, $\alpha_{i}$ sâo linearmente independentes sobre $K_{y}$ isto implica em $\mu_{o} \leq d$.

Portanto, $\mu_{a}=d$.

Assim, existem funções $A_{o}, \ldots, A_{\mu_{a}-1}$ pertencentes a $\mathcal{O}_{y}$ tais que

$$
p\left(z_{1}\right)=z_{1}^{\mu_{a}}+\sum_{j=0}^{\mu_{a}-1} A_{j}\left(\frac{\partial f}{\partial z_{1}}, \ldots, \frac{\partial f}{\partial z_{n}}\right) z_{1}^{j}=0
$$

Agora, sejam $a$ e $b$ pontos distintos tais que $\partial f(a)=\partial f(b)=y$. Consideremos a função coordenada $z_{j}(z)=z_{j}$. Temos que cada $z_{j}$ pertence a $K_{z}$, logo

$$
z_{j}(z)=z_{j}=\sum_{k} \Gamma_{j k}(\partial f(z)) z_{1}(z)^{k}, \text { para } j=2, \ldots, n
$$

Afirmamos que $z_{1}(a) \neq z_{1}(b)$. De fato, suponhamos que $z_{1}(a)=z_{1}(b)$. Como $a \neq b$ existe um $j$ tal que $z_{j}(a) \neq z_{j}(b)$ mas,

$$
z_{j}(a)=a_{j}=\sum_{k} \Gamma_{j k}(\partial f(a)) z_{1}(a)^{k}=\sum_{k} \Gamma_{j k}(\partial f(b)) z_{1}(b)^{k}=z_{j}(b)=b_{j}
$$


o que é uma contradição.

Pelo Teorema 2.4, para $y$ suficientemente próximo de 0 , valor regular de $\partial f$ a equaçào $\partial f(z)-y=0$ possui exatamente $\mu_{g}$ soluşôes cada uma delas dando origem a uma solução distinta da equação polinomial

$$
z_{1}^{\mu_{a}}+\sum_{j=0}^{\mu_{a}-1} A_{j}(y) z_{1}^{j}=0
$$

Logo, $\mu_{g} \leq \mu_{a}$.

Por outro lado olhando para a subvariedade analítica de $\mathbf{C}^{n+1}$ definida por

$$
\left\{\left(y_{1}, \ldots, y_{n}, z_{1}\right): z_{1}^{\mu_{a}}+\sum_{j=0}^{\mu_{a}-1} A_{j}(y) z_{1}^{j}=0\right\}
$$

Ainda, se $y$ é tal que existem $\mu_{a}$ soluçôes para a equaçâo, então existem $\mu_{a}$ pontos distintos na pré-imagem de $y$ por $\partial f$, isto porque fixados $z_{1}$ e $y$ existe um único ponto $\left(z_{1}, z_{2}\left(z_{1}, y\right), \ldots, z_{n}\left(z_{1}, y\right)\right)$ tal que $\partial f\left(z_{1}, z_{2}\left(z_{1}, y\right), \ldots, z_{n}\left(z_{1}, y\right)\right)=y$, $\log 0 \mu_{a} \leq \mu_{g}$.

Portanto $\mu_{a}=\mu_{g}$. 


\section{Capítulo 5}

\section{Cálculo do Número de Milnor}

O objetivo deste capitulo é calcular o número de Milnor, $\mu$, de germes quasehomogêneos, tendo na origem uma singularidade isolada.

O resultado principal, Teorema 5.1 é devido a Milnor e Orlik [4].

Definição 5.1 Sejam $w_{1}, \ldots, w_{n}$ números racionais positivos. Um polinômio $f\left(z_{1}, \ldots, z_{n}\right)$ é dito quase-homogênto do tipo $\left(w_{1}, \ldots, w_{n}\right)$ se ele pode ser expresso como combinaçáo linear de monómios $z_{1}^{i_{1}} \ldots z_{n}^{i_{n}}$ tal que

$$
\frac{i_{1}}{w_{1}}+\ldots+\frac{i_{n}}{w_{n}}=1
$$

Lema 5.1 Se uma aplicação polinomial $G: \mathrm{C}^{n} \rightarrow \mathrm{C}^{n}$ é tal que sua i-ésima componenté é um polinômio homogêneo de grau $d_{i}, \epsilon$ se $G^{-1}(0)=0$ entâo o grau local de $G \in \mathrm{\epsilon m} O$ é igual a $d_{1} \ldots d_{n}$.

\section{Demonstração:}

As hipersuperficies $G_{i}^{-1}$ estendem-se a hipersuperfícies projetivas $H_{i} \subset \mathbf{P}^{n}(\mathbf{C})$ de mesmo graus. A multiplicidade de interseç̧âo de $H_{1}, \ldots, H_{n}$ é igual ao produto $d_{1} \ldots d_{n}$ dos graus. Mas a intersecção $H_{1} \cap \ldots \cap H_{n}$ claramente consiste de um único ponto $0 \in \mathbf{C}^{n} \subset \mathbf{P}^{n}\left(\mathbf{C}^{n}\right)$. 
Teorema 5.1 Seja $f\left(z_{1}, \ldots, z_{n}\right)$ um polinômio quase-homogêneo do tipo $\left(w_{1}, \ldots, w_{n}\right)$, tendo na origem uma singularidade isolada. Entâo

$$
\mu=\left(w_{1}-1\right)\left(w_{2}-1\right) \ldots\left(w_{n}-1\right)
$$

\section{Demonstração:}

Expressemos cada peso $w_{i}$, como fraçâo irredutivel $w_{i}=u_{i} / v_{i}, \quad i=1, \ldots, n$.

Seja $d=\left[u_{1}, \ldots, u_{n}\right]$ o mínimo múltiplo comum dos $u_{i}$ e $q_{i}=d / w_{i}, \quad i=1, \ldots, n$.

Dado $G\left(z_{1}, \ldots, z_{n}\right)=\left(z_{1}^{q_{1}}, \ldots, z_{n}^{q_{n}}\right)$, temos que $f \circ G$ é homogêneo de grau $d$, pois como $f$ é combinação linear de monômios $z_{1}^{i_{1}}, \ldots, z_{n}^{i_{n}}$, o grau de cada parcela de $f \circ G$ é dado por

$$
i_{1} q_{1}+\ldots+i_{n} q_{n} \doteq i_{1} \frac{d}{w_{1}}+\ldots+i_{n} \frac{d}{w_{n}}=d\left(\frac{i_{1}}{w_{1}}+\ldots+\frac{i_{n}}{w_{n}}\right)=d
$$

Logo cada i-ésima componente de $\partial f \circ G$ é um polinômio homogêneo de grau $d-q_{i}$. De fato, temos que o grau de cada parcela da i-ésima componente de $\partial f \circ G$ é dado por

$$
i_{1} q_{1}+\ldots+\left(i_{i}-1\right) q_{i}+\ldots+i_{n} q_{n_{i}}=d\left(\frac{i_{1}}{w_{1}}+\ldots+\frac{i_{n}}{w_{r_{1}}}\right)-q_{i}=d-q_{i}
$$

Portanto, pelo Lema 5.1, grau $(\partial f \circ G)=\left(\dot{d}-q_{1}\right) \ldots\left(d-q_{n}\right)$. Por outro lado $\operatorname{grau}(\partial f \circ G)=\operatorname{grau}(\partial f) \operatorname{grau}(G)$ portanto,

$$
\mu_{g}(f)=\operatorname{grau}(\partial f)=\frac{\left(d-q_{1}\right)}{q_{1}} \ldots \frac{\left(d-q_{n}\right)}{q_{n}}=\left(w_{1}-1\right) \ldots\left(w_{n}-1\right)
$$

como queríamos.

Exemplo 5.1 Os polinômios de Brieskorn

$$
f\left(z_{1}, \ldots, z_{n}\right)=z_{1}^{a_{1}}+z_{2}^{a_{2}}+\ldots+z_{n}^{a_{n}}
$$

são quase-homogêneos do tipo $\left(a_{1}, \ldots, a_{n}\right)$.

$$
\text { Portanto, } \mu=\left(a_{1}-1\right)\left(a_{2}-1\right) \ldots\left(a_{n}-1\right) \text {. }
$$


Exemplo 5.2 Seja $f(z, w)=z^{2}+w^{5}$. Temos que $f$ é um polinômio quasehomogêneo do tipo $(2,5)$.

$$
\text { Portanto, } \mu=(2-1)(5-1)=4 \text {. }
$$

Exemplo 5.3 Seja $f(z, w)=z^{2} w+w^{4}$. Temos que $f$ é um polinômio quasehomogêneo do tipo $(8 / 3,4)$.

$$
\text { Portanto, } \mu=(8 / 3-1)(4-1)=5 \text {. }
$$




\section{Bibliografia}

[1] MILNOR, J. [1968]. Singular Points of Complex Hypersurfaces. Annals of Math. Studies, 61, Princeton.

[2] MILNOR, J. [1963]. Morse Theory. Annals of Math. Studies, 51, Princeton.

[3] MILNOR, J. [1965]. Topology from the Differentiable Viewpoint. The University Press of Virginia.

[4] MILNOR, J.; ORLIK, P. [1970]. Isolated Singularities Defined by Weighted Homogeneous Polynomials. Topology, vol. 9, pp. 385-393 Pergamon Press. Printed in Great Britain.

[5] CARNEIRO, M. J. D.; SOARES, M. G. [1985]. Introdução à Topologia de Singularidades Complexas. 15o Colóquio Brasileiro de Matemática, IMPA.

[6] GIBSON, C. G. [1979]. Singular Points of Smooth Mappings. Research Notes in Mathematics, 25. London, Pitman.

[7] GOLUBITSKY, M.; GUILLEMIN, V. [1973]. Stable Mappins and their Singularities. Graduate Texts in Mathematics, 14. Springer-Verlag, New York.

[8] SPANIER, E. [1966]. Algebraic Topology. McGraw-Hill.

[9] GUILlemin, V.; POLlACK, A. [1974]. Differential Topology. PrenticeHall, Inc., Englewood Cliffs, New Jersey.

[10] MASSEY, W. S. [1977]. Algebraic Topology: An Introduction. Graduate Texts in Mathematics, 56. Springer-Verlag, New York.

[11] MAC LANE, S. [1963]. Homology. G.M.W.,114. Springer-Verlag, Berlin.

[12] GRIFFITHS, P.; HARRIS, J. [1978]. Principles of Algebraic Geometry. John Wiley \& Sons Inc.

[13] LANG, S. [1965]. Algebra. Addison-Wesley, Publishing Company. 
[14] NAGATA, M. [1975]. Local Rings. Robert E. Krieger Publishing Company Huntington, New York.

[15] STEENROD. N. [1951]. The Topology of Fibre Bundles. Princeton University Pres.

[16] LIMA, E. L. [1977]. Grupo Fundamental e Espaços de Recobrimento. 119 Colóquio Brasileiro de Matemática, IMPA, Poços de Caldas.

[17] TRÁNG, L. D. [1973]. Topologie des Singularites des Hypersurfaces Complexts. Asterisque 7 e 8 , pp. 171-182.

[18] TRÁNG, L. D.; RAMANUJAM, C. P. [1976]. The Invariance of Milnor's Number Implies the Invariance of the Topological Type. American Journal of Mathematics, vol. 98, № 1, pp. 67.78.

[19] TIMOURIAN, J. G. [1977]. The Invariance of Milnor's Number Implies the Topological Triviality. American Journal of Mathematics, vol. 99, № 2, pp.437-446.

[20] MOND, D. [1987]. Somes Remarks on the Geometry and Classification of Germs of Maps from Surfaces to 3-spact. Topology 26, pp. 361-383.

[21] MARAR, W: L.; MOND, D. [1989]. Multiple Point Schemes for Corank 1 Maps. Journal of the London Mathematical Society (2) 39, pp. 553-567. 